\title{
Deficits in Visual Motion Processing Following Ibotenic Acid Lesions of the Middle Temporal Visual Area of the Macaque Monkey ${ }^{1}$
}

\author{
WILLIAM T. NEWSOME, ${ }^{2}$ ROBERT H. WURTZ, ${ }^{3}$ MAX R. DÜRSTELER, AND AKICHIKA MIKAMI ${ }^{4}$
}

National Institutes of Health, National Eye Institute, Laboratory of Sensorimotor Research, Bethesda, Maryland 20205

\begin{abstract}
Physiological experiments have produced evidence that the middle temporal visual area (MT) of the monkey is selectively involved in the analysis of visual motion. We tested this hypothesis by studying the effects of small chemical lesions of MT on eye movements made in response to moving as opposed to stationary visual targets. We observed two deficits for eye movements made to moving targets: a monkey's ability to match the speed of his smooth pursuit eye movements to the speed of the moving target was impaired, and a monkey's ability to adjust the amplitude of a saccadic eye movement to compensate for target motion was impaired. In contrast, saccades to stationary targets were unaffected by the MT lesions, suggesting that monkeys with MT lesions had more difficulty responding to moving than to stationary stimuli. These results provide the first behavioral evidence that neural processing in MT contributes to the cortical analysis of visual motion.
\end{abstract}

Primate extrastriate visual cortex is composed of a remarkable array of distinct visual areas. In the macaque monkey no fewer than nine extrastriate areas have been identified on the basis of anatomical connections, visual topography, cortical architectonics, and single neuron response properties (for reviews, see Zeki, 1978; Van Essen, 1979; Woolsey, 1981). How these areas individually and collectively contribute to vision is a central issue for a neurobiological understanding of visual processing.

Perhaps the most intensively studied of these areas is the middle temporal visual area (MT), an area in the posterior bank of the superior temporal sulcus (STS) which receives a direct projection

Received July 5, 1984; Revised September 20, 1984; Accepted September 24, 1984

\footnotetext{
${ }^{1}$ We are grateful to Dr. S. G. Lisberger, who suggested the utility of pursuit initiation as a probe for visual motion processing and who made several contributions to the design of these experiments. We thank Dr. L. M. Optican for providing the original version of the data analysis program. Drs. M. E. Goldberg, J. H. R. Maunsell, and D. C. Van Essen provided helpful suggestions during the preparation of this manuscript. We also thank George Cresswell and Laura Cooper for excellent histological assistance, and Pamela Brown and Jean Steinberg for typing the manuscript. M. R. D. was supported by Schweizerische Stiftung für medizinisch-biologische Stipendien.

${ }^{2}$ Present address: Department of Neurobiology and Behavior, State University of New York, Stony Brook, NY 11794.

${ }^{3}$ To whom correspondence should be addressed, at Laboratory of Sensorimotor Research, National Eye Institute, Building 10, Room 6C420, Bethesda, MD 20205

${ }^{4}$ Present address: Department of Neurophysiology, Primate Research Institute, Inuyama 484 , Japan.
}

from striate cortex. The anatomical identification of this area in the macaque was first reported by Zeki (1969) and Cragg (1969). Its boundaries and visual topography were determined in the owl monkey by Allman and Kaas (1971), who named the region the middle temporal area, a term we will use throughout this report. MT has now been identified in several other primates (Spatz and Tigges, 1972, 1973; Allman et al., 1973; Tigges et al., 1981) and appears to be a homologous structure in all of these species (Van Essen et al., 1981). MT has proven to be attractive for study, in part because its boundaries can be determined from its distinctive myeloarchitecture, its visual topography has been mapped, and its anatomical connections are known (Ungerleider and Mishkin, 1979; Gattass and Gross, 1981; Van Essen et al., 1981; Ungerleider et al., 1982; Maunsell and Van Essen, 1983c; Standage and Benevento, 1983; Weller and Kaas, 1983; Weller et al., 1984).

An initial report by Dubner and Zeki (1971) and subsequent studies by Zeki $(1974 a, b)$ established that MT contains a preponderance of neurons which encode the direction of motion of visual stimuli, and this observation led to the hypothesis that MT plays a specific role in the analysis of visual motion. MT neurons have now been found to be selective for speed and binocular disparity as well as for direction (Baker et al., 1981; Maunsell and Van Essen, 1983a, b), and a columnar system of direction representation has been reported (Albright et al., 1984). Neurons in owl monkey MT have direction- and speed-selective surround mechanisms which may enable certain MT neurons to distinguish figure from ground on the basis of relative motion (Miezin et al., 1982), and recent work in our laboratory suggests that MT neurons play a role in the perception of apparent motion (Mikami et al., 1983; Wurtz et al., 1984a). Perhaps most interesting are recent experiments which show that some MT neurons respond to the unitary motion of two-dimensional patterns. in contrast to neurons in striate cortex which respond to the motion of the oriented components of such patterns (Gizzi et al., 1983; Movshon et al., 1984). Such studies, of single cell responses of MT have provided a wealth of information that is consistent with the original hypothesis that MT is specialized for the analysis of visual motion.

Although MT is the extrastriate area for which we have the clearest ideas concerning function, these hypotheses have been completely dependent upon inference from single neuron response properties. Such hypotheses could be tested by assessing the behavioral consequences of removal or inactivation of MT. Our goal in this study has been to acquire direct behavioral evidence concerning the significance for vision of neural processing in MT. Our central strategy was to examine the effects of small chemical lesions of MT on a monkey's performance in a motion-dependent behavioral task-the initiation of smooth pursuit eye movements. Pursuit initiation seemed an appropriate choice for our purposes since the monkey's task was to match the speed and direction of its smooth eye movements to the speed and direction of a moving visual target. Such responses clearly require accurate information concerning target motion and may, therefore, be dependent upon visual motion 
processing in MT. The importance of parieto-occipital cortex for smooth pursuit is also suggested by clinical observations (Troost et al., 1972; Troost and Abel, 1982; Zihl et al., 1983) and surgical ablations in monkcys which reveal deficits in pursuit eye movements (Goldberg et al., 1982; Lynch and McLaren, 1982; Zee et al., 1982). The chemical lesions were created with ibotenic acid, a neurotoxin which appears to affect cell bodies selectively, leaving fibers of passage unharmed (Schwarcz et al., 1979; Guldin and Markowitsch, 1981). The ibotenic acid lesions were small, and they affected only a restricted region of the contralateral visual field. Nonaffected regions of the contralateral field as well as the entire ipsilateral field provided concurrent, within-animal controls.

Our results show that eye movements made in response to moving targets are deficient following MT lesions, whereas those to stationary stimuli are completely unaffected. The behavioral deficits appear to result from disruption of visual motion processing rather than damage to eye movements per se.

A brief report of these results has appeared previously (Newsome et al., 1983)

\section{Materials and Methods}

Three methodological steps were involved in these experiments: training of monkeys on the behavioral task, physiological identification of MT and injection of ibotenic acid, and quantitative analysis of the behavioral data.

Behavioral task. Under general anesthesia (sodium pentobarbital), monkeys were implanted with a head holder for restraint of the head during the experiments, with a stainless steel cylinder for microelectrode rccording (Evarts, 1966, 1968), and with an eye coil for measurement of eye position (Judge et al., 1980). A detailed description of these procedures can be found in Hikosaka and Wurtz (1983). Following surgery, monkeys were giver analgesics for several days and were allowed to recover for at least a week before training began

The three monkeys used in these experiments (Macaca mulatta, female 4 to $6 \mathrm{~kg}$ ) were trained to fixate or to follow with their eyes a small visual target presented on a tangent screen $86 \mathrm{~cm}$ in front of them. Each monkey was seated in a primate chair with the head restrained so that the eyes, when in primary position, were directed toward the center of the screen. The monkeys sat inside magnetic field coils with diameters of 3 feet and had an unobstructed view of the screen out to $40^{\circ}$ eccentricity. Eye movements were recorded using the magnetic search coil technique of Robinson (Robinson, 1963; Fuchs and Robinson, 1966).

Target presentation on the tangent screen was confined to the horizontal meridian to simplify data analysis. A trial was initiated by turning on a small light at the center of the screen which the monkey had to fixate for a variable length of time (Fig. $1 A$ ). When this light was extinguished, the monkey moved its eyes to a second light which appeared simultaneously at one of several possible locations on the horizontal meridian. In "step" trials (Fig. 1B), the second target remained stationary and the monkey made a rapid (saccadic) eye movement to the target. In "step-ramp" trials, the second target moved at $16^{\circ} / \mathrm{sec}$ away from (Fig. $1 \mathrm{C}$ ) or toward (Fig. $1 D$ ) primary position along the horizontal meridian. On these trials the monkey acquired the target with its fovea and pursued the target with smooth eye movements for the duration of the trial. The target "step" for a given trial was usually $2^{\circ}, 5^{\circ}, 10^{\circ}$, or $15^{\circ}$ in amplitude and could occur in either horizontal direction from the primary position (Fig. 1A). The size and direction of the target step, the direction of target motion following the step, and the trial condition ("step" or "step-ramp") were randomized from trial to trial. We chose $16^{\circ} / \mathrm{sec}$ for target speed because the aggregate output of MT neurons is maximal at this speed (Maunsell and Van Essen, 1983a)

A block of trials usually contained all 24 different trial types: 8 "step" locations along the horizontal meridian and 3 conditions for each "step" location (step, step-ramp away, and step-ramp toward). Trial presentations continued within a block until the monkey successfully initiated 10 trials for each trial type (240 correct trials). When the monkey completed 10 trials for one trial type, it was dropped from the table and ensuing trials were randomly selected from those types remaining. The step-ramp paradigm was used so that we could instantaneously place a moving stimulus in a particular part of the visual field. By carefully analyzing the monkey's saccade and initial pursuit movement, we were able to assess its ability to utilize motion information obtained from the visual field locus to which the target "stepped" (see "Results"). The range of step locations illustrated in Figure $1 A$ was employcd in order to exploit the retinotopic organization of MT. Our small lesions affected a restricted portion of the visual field, so that test locations
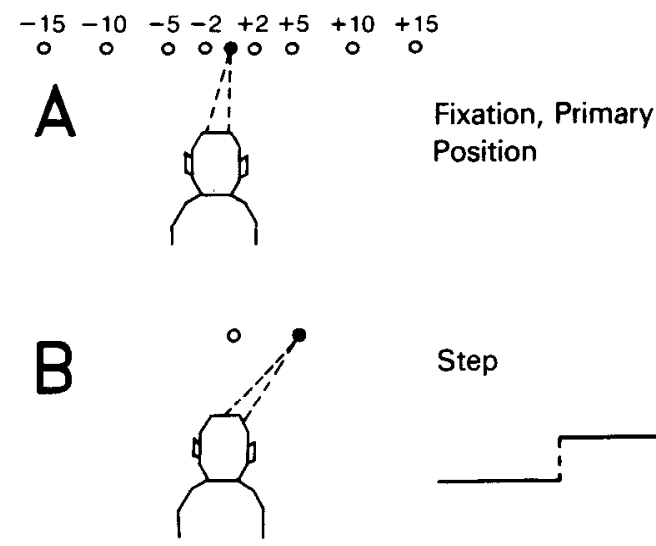

Step

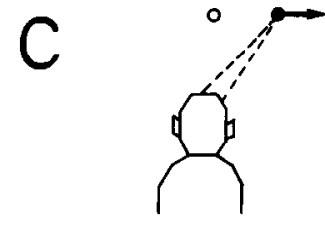

Step-Ramp, Away

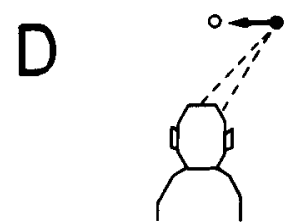

Step-Ramp, Toward

Figure 1. Behavioral paradigm. A, All trials were initiated by the appear ance of a fixation target at primary position (solid circle). After the monkey fixated this light for a variable length of time, it was extinguished and a second target appeared at one of several possible locations on the horizontal meridian (open circles). When the second target appeared, one of three trial types occurred as in the lower panels. $B$, In some trials the second light remained stationary ("step" trial), in which case the monkey made a saccadic eye movement and fixated the second target until the end of the trial. $C$ and $D$, In the remaining trials the second target moved at $16 \%$ sec along the horizontal meridian away from (C - "step-ramp, away" trial) or toward $(D-$ "step-ramp, toward" trial) primary position. In these trials the monkey acquired the target, generally with a saccade, and then tracked the target with smooth pursuit eye movements for the duration of the trial.

outside the affected portion of the field served as controls for the influence of extraneous factors on the monkey's behavior.

During the initial training period, monkeys were rewarded for fixating a spot of light and detecting the dimming of that spot (Wurtz, 1969), but throughout the experimental procedures this task was used only to calibrate the eye position at the beginning of each experimental day. For the actual experiments the monkeys were rewarded with a drop of water for keeping their eyes inside an electronically defined "window" centered on the target (Sparks et al., 1976). At the beginning of each trial, the fixation light came on and the monkey was required to move its eye into the position window around the fixation light within $500 \mathrm{msec}$. This fixation window was kept small to minimize differences in fixation position between trials (usually \pm 0.7 to $\pm 1.5^{\circ}$ ). The fixation light remained on for 500 to $2500 \mathrm{msec}$ (after the monkey's eye entered the window) and was then extinguished as the target light came on. The monkey was allowed $400 \mathrm{msec}$ to shift its gaze to the target light. After this time the position window was centered on the target and moved with the target, and the monkey was rewarded if it maintained eye position within that window until the end of the trial $(500$ to $800 \mathrm{msec})$ During pursuit of the moving target, the position window varied in size $\left( \pm 1.0^{\circ}\right.$ to $\left.\pm 5.0^{\circ}\right)$ from trial to trial, depending upon the eccentricity of the target on the screen and the consequent difficulty for the monkey. The window size was constant for a particular trial type before and after the lesion. Window sizes were large enough so that changes in the response following the lesion did not result in increased frequency of missed trials.

The visual target was relatively small $\left(0.2^{\circ}\right.$ in diameter) and bright $\left(38 \mathrm{~cd} / \mathrm{m}^{2}\right)$ against a dim background $\left(0.13 \mathrm{~cd} / \mathrm{m}^{2}\right)$. The target was produced 
by projecting a spot of light from a tungsten filament bulb onto mirrors mounted on galvanometers. The fixation point was a small spot $\left(0.05^{\circ}\right.$ in diameter) produced by projecting a beam onto the screen from a lightemitting diode.

Throughout the training and the experiments, the monkey's weight was checked each day and supplementary water or fruit was provided if needed. Monkeys sat in a primate chair during experiments but were returned to their home cages each day after the experimental session.

Injection of ibotenic acid. MT was first located using glass-insulated platinum microelectrodes (Wohlbarsht et al., 1960) advanced into the STS in a parasagittal plane with the electrode angled roughly $20^{\circ}$ above horizontal. Electrodes passed through striate cortex and the lunate sulcus before entering MT. While the monkey fixated a spot of light, the receptive fields of cells throughout the penetration were located and roughly characterized. MT was identified on the basis of its location within the STS, the characteristic direction selectivity of its neurons, and the relatively restricted size of its receptive fields (compared to those of areas deeper in the sulcus). Following localization of MT, a 19 gauge quide tube was implanted within the recording cylinder so that the tip of the tube was within $3 \mathrm{~mm}$ of MT. One end of the guide tube was beveled so as to be easily introduced through the dura, and the guide tube was anchored to the inner wall of the cylinder using dental acrylic cement. Insertion of the guide tube was performed while the monkey was under ketamine hydrochloride $(0.3 \mathrm{ml}$ of $100 \mathrm{mg} / \mathrm{ml})$. We applied antibiotic ointment to the top of the guide tube and closed it with a stainless steel insert between experiments.

We placed the guide tube so that the representation of the horizontal meridian in MT was accessible through the guide tube. We confirmed the guide tube location by recording neuronal receptive fields with a fine tungsten electrode (Haer) introduced through the guide tube. We then inserted through the guide tube the needle of a $1-\mu$ l syringe (Hamilton) which had been insulated to allow recording of multiple cell activity. This arrangement permitted precise control of the site of the injection and allowed us to record the visual field location where the ibotenic acid was released. We injected $1 \mu \mathrm{l}$ of ibotenic acid $(15 \mathrm{mg} / \mathrm{ml}$ in a basic saline solution). Monkeys showed no signs of discomfort during or at any point after the injection, and behavioral testing could be resumed immediately.

Following completion of experiments, monkeys were deeply anesthetized and perfused with saline and $10 \%$ formalin. Parasagittal sections through the occipital and parietal lobes were stained with cresyl violet for cell bodies and with a modified silver stain (Gallyas, 1979) for myelinated fibers. The extent of the lesions was determined by examination of the histological sections, and the data were displayed on "unfoided" maps of the cortex created by the method of Van Essen and Maunsell (1980).

Data analysis. The behavioral tasks as well as storage and display of data were controlled by a real-time experimental system (REX) developed by Hays et al. (1982) which was run on a PDP 11/40 computer. We sampled and stored horizontal and vertical eye position and mirror position on each trial for a period, lasting about $1 \mathrm{sec}$, which began $150 \mathrm{msec}$ before target onset. The horizontal and vertical eye position signals were low pass filtered at 250 $\mathrm{Hz}$ and then digitized at $500 \mathrm{~Hz}$. The entire system had a resolution of $0.1^{\circ}$.

For quantitative analysis of the eye movements, we used an off-line program which automatically identified and measured relevant features of the smooth pursuit response and saccadic eye movements within individual trials. This program computed velocity and acceleration of the eye from the stored position information using a finite response digital filter with linear phase and $30 \mathrm{~Hz}$ bandpass (Rabiner and Gold, 1975). The experimenter then specified certain velocity and acceleration criteria by which the program automatically identified the beginning and the end of saccadic eye movements in each trial in the data file. These criteria were initially selected so that the identification of saccadic and pursuit eye movements looked reasonable to the experimenter; once selected, the same criteria were used for analysis of all of our data. Once a trial had been automatically marked, each record with its marks was displayed on a video monitor (of which Fig. 2 is an example) and visually inspected by an experimenter.

In each record, the occurrence of a saccade was indicated when eye acceleration exceeded $750^{\circ} / \mathrm{sec}^{2}$. After a saccade was identified, the maximum eye acceleration was located, and the program then moved back in time through the data buffer until a point was reached at which eye acceleration was $80 \%$ of the maximum. This point was marked as the beginning of the saccade ( $s$ in Fig. 2). The end of the saccade was marked by a similar procedure. The program skipped forward $70 \mathrm{msec}$ through the buffer to get beyond the end of the saccade and then repeated the process by which it identified the beginning of the saccade, except that the movement through the buffer was reversed in direction. The end of the saccade was marked with a letter $r$ as in Figure 2.

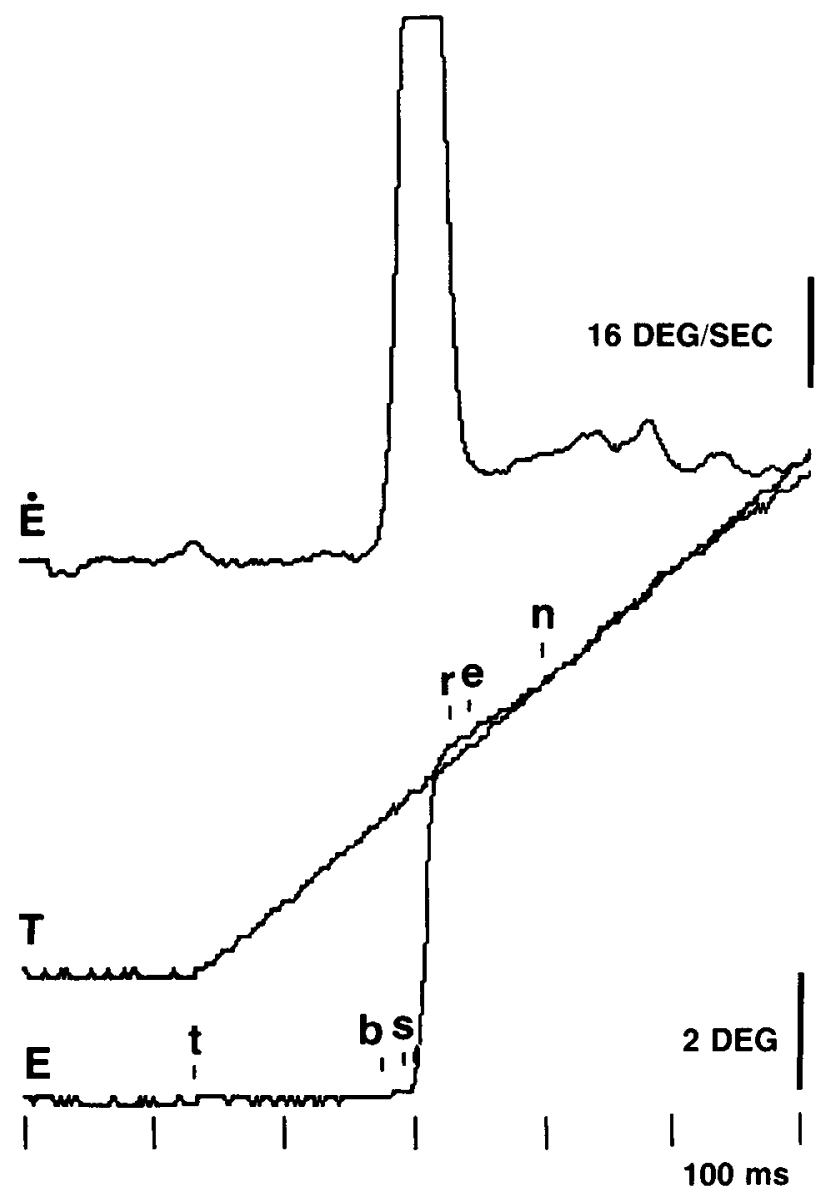

Figure 2. Illustration of a single pursuit trial as automatically "marked" by a computer program for quantitative analysis. $E$ indicates horizontal eye position as recorded during the experiment, $T$ shows the position of the tracking target as recorded during the experiment, and $\dot{E}$ shows horizontal eye velocity as determined by digital differentiation of the eye position trace. All three quantities are plotted against time as indicated by the scale at the bottom. $t$, target onset; $b$, beginning of saccade in velocity trace; $s$, beginning of saccade in position trace; $r$, end of saccade in position trace; $e_{\text {, end of }}$ saccade in velocity trace; $n$, point $70 \mathrm{msec}$ after end of initial saccade at which smooth eye speed was measured.

TABLE I

Summary of ibotenic acid injections

\begin{tabular}{cccl}
\hline Experiment & Monkey & Hemisphere & \multicolumn{1}{c}{ Manipulation } \\
\hline B1 & B & Left & Ibotenic acid in MT \\
C1 & C & Right & Ibotenic acid in MT \\
D0 & D & Left & Normal saline in MT \\
D1 & D & Left & Ibotenic acid in MT \\
D2 ${ }^{a}$ & D & Right & Ibotenic acid in MT \\
\hline
\end{tabular}

${ }^{a}$ The control hemifield for this injection had previously served as the test hemifield for injection D1. Several weeks of recovery permitted return to prelesion performance in this hemifield before injection D2 was made.

Most measurements of smooth eye speed presented in the data figures were made $70 \mathrm{msec}$ after the end of the initial saccade ( $n$ in Fig. 2). However for one animal (D), occasional "catch-up" saccades occurred with latencies of 70 to $100 \mathrm{msec}$ after the initial saccade. To avoid contamination of pursuit eye speed measurements by these "catch-up" saccades, eye speed was measured $50 \mathrm{msec}$ after the initial saccade for this experiment. In figures such as Figure $3 B$, in which smooth eye speed is continuously displayed as a function of time, we excised any interval of the velocity trace which was affected by a saccade so that smooth eye speed could be viewed in isolation. We could not depend on the $s$ and $r$ marks as a guide in this process since the $30-\mathrm{Hz}$ bandpass characteristic of our digital filter "spread" the effect of the saccade in the velocity trace (see Fig. 2). Our program 

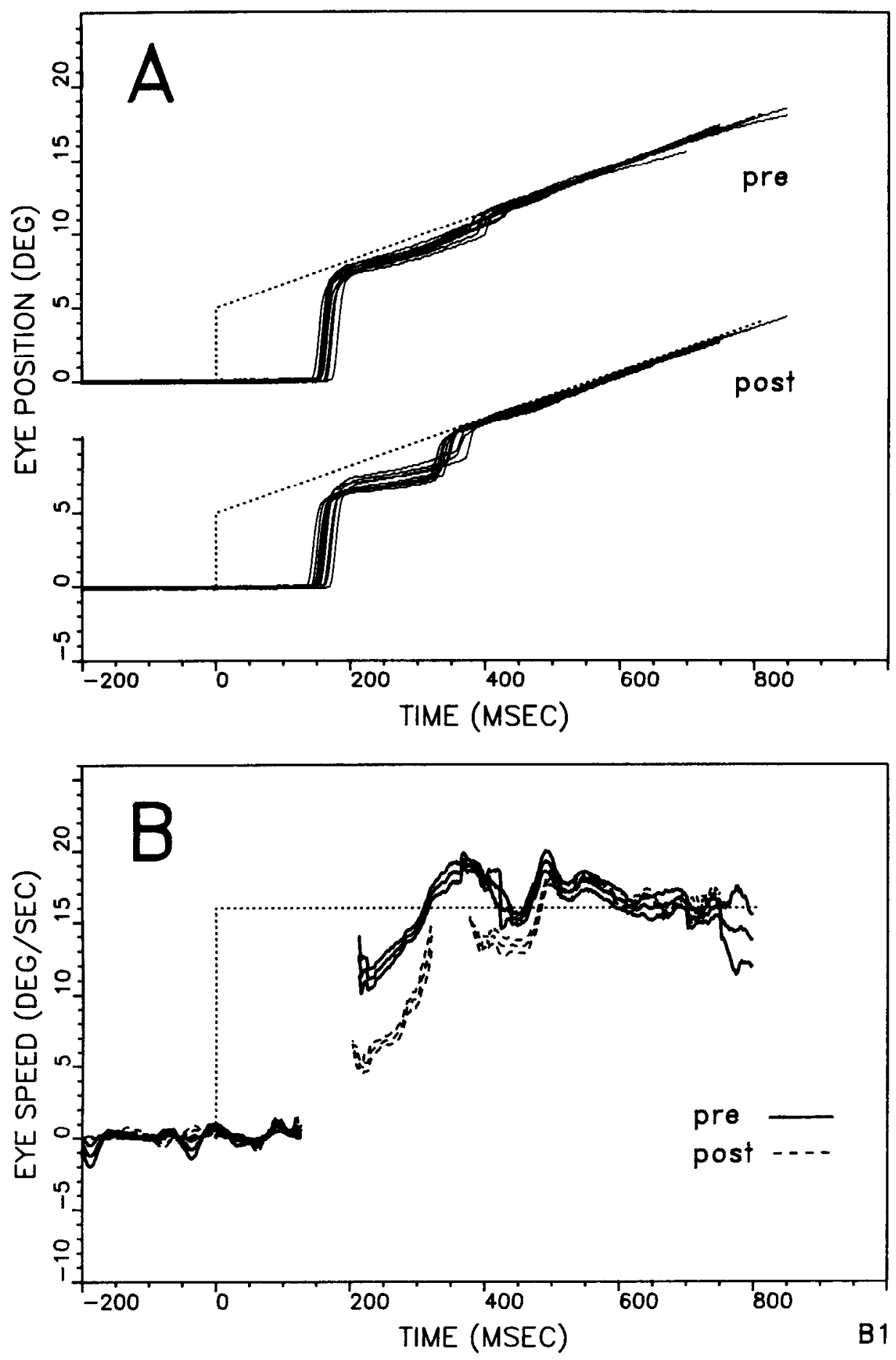

Figure 3. Deficit in pursuit initiation following an ibotenic acid lesion of MT. $A$, Horizontal eye movements made in response to a pursuit target which underwent a rightward "step-ramp" displacement. Time is represented on the $a b$ scissa, and horizontal eye position is shown on the ordinate. The target stepped $5^{\circ}$ to the right and then moved smoothly to the right at $16^{\circ} \%$ sec (dashed line). The top set of traces shows 10 superimposed responses recorded before an injection of iboteric acid into MT. The bottom set of traces shows impaired responses of the same monkey $24 \mathrm{hr}$ after the injection. $B$, Mean smooth eye speed (with standard error) is plotted as a function of time for the pre- and postlesion traces illustrated in $A$. Target speed is shown by the dashed line; saccades have been excised. Postlesion eye speed is significantly lower than prelesion speed for the first $100 \mathrm{msec}$ following the initial saccade. The low amplitude "ringing" occasionally visible in the velocity traces in this and subsequent figures is noise introduced by the digital filter. The results were not unduly affected by such noise. The label (B1) in the lower righthand comer of this and subsequent figures indicates the experiment in which these data were recorded. These labels may be cross-referenced with the capsule summary of experiments in Table I. For figures which contain data from more than one experiment, an appropriate label appears at the lower righthand corner of each panel. therefore made two more marks ( $b$ and $e$ in Fig. 2) which indicated the beginning and end of the saccade in the velocity trace and marked the interval for excision. These marks were placed at the points where eye acceleration reached $750^{\circ} / \mathrm{sec}^{2}$. Visual inspection of Figure 2 indicates that the bandpass of the digital filter did not result in contamination by the saccade of our smooth eye speed measurement at mark $n$.

Records were rejected only for the following reasons: the saccade to the target occurred with a latency of less than $100 \mathrm{msec}$, indicating that the saccade was either an "express" saccade (Fischer and Boch, 1983) or was made in anticipation of, rather than in response to, the target; the monkey did not, in the expermenter's judgment, make a reasonable attempt to track the target: or noise or artifact occurred on the eye movement record. An average of $5 \%$ of the trials in any given block was rejected for these reasons.

\section{Results}

The results described here are from four injections of ibotenic acid in three monkeys, and a capsule summary of each injection is given in Table I. The monkey's eye movements were deficient in two ways following ibotenic acid lesions of MT: eye speed was poorly matched to target speed during pursuit initiation, and the amplitude of the initial saccade did not compensate for the motion of the target. We will consider each of these deficits in turn.

Speed matching. Figure 3 illustrates the effects of an MT lesion on pursuit initiation. Ten normal pursuit responses to one of the "step-ramp" stimuli obtained before injection B1 are superimposed in the upper set of traces in Figure $3 A$. In these trials the target appeared $5^{\circ}$ to the right of primary position and moved away from primary position at $16^{\circ} / \mathrm{sec}$. The monkey's response had two major components. First, the monkey made a saccadic eye movement (latency $\cong 150 \mathrm{msec}$ ) which brought the fovea near the target. Second, the monkey pursued the target with smooth eye movements 
for the duration of the trial. In most trials a small "catch-up" saccade was necessary to bring the fovea directly onto the target.

The important point to note is that the monkey established pursuit in the appropriate direction and with a roughly appropriate speed within a few tens of milliseconds of the end of the initial saccade. Since the shortest pursuit latencies reported in the literature are around 80 msec (Lanman et al., 1978), the monkey's pursuit in the first $80 \mathrm{msec}$ after the initial saccade must depend upon information about target motion derived from the peripheral retina before the saccade. Thus, in the example of Figure $3 A$, the first $80 \mathrm{msec}$ of postsaccadic pursuit is in response to visual information obtained while the target was moving within a region 5 to $7^{\circ}$ eccentric in the right hemifield. By varying the size and direction of the "step" and measuring eye speed within $80 \mathrm{msec}$ of the end of the saccade, we were therefore able to test the effects of an MT lesion on the monkey's use of motion information from various locations in the visual field.

The lower set of traces in Figure $3 A$ shows the monkey's responses to the same stimulus $24 \mathrm{hr}$ after an injection of ibotenic acid into MT. The injection was made on the representation of the horizontal meridian at an eccentricity of $6^{\circ}$ (left hemisphere, right hemifield). The most striking change in the pursuit response was a reduction in eye speed during the first $100 \mathrm{msec}$ of postsaccadic pursuit (note the reduced slope of the eye position traces). This deficit is more easily evaluated by examination of the composite eye speed data displayed in Figure $3 B$. Mean eye speed and the standard error of the mean are plotted against time for the pre- and postlesion traces illustrated in Figure $3 A$. Postlesion eye speed was reduced to about $50 \%$ of prelesion levels immediately following the initial saccade. Eye speed reached normal levels 100 to $200 \mathrm{msec}$ following the saccade, presumably because accurate information about the motion of the target was obtained from an uncompromised portion of the hemifield after the saccade was made. Note that the deficit does not reflect a motor inability to perform smooth eye movements: after a corrective saccade, the monkey pursued the target in a normal fashion (Fig. $3 A$, lower traces). Thus, only the initial interval of postsaccadic pursuit, which was dependent upon visual motion at an eccentricity of 5 to $7^{\circ}$ in the right hemifield, was impaired.

A similar analysis was made for all pursuit trials in this experiment, and Figure $4 A$ shows the mean and standard error of eye speed measured $70 \mathrm{msec}$ after the initial saccade for step-ramp trials with steps to eight different locations on the horizontal meridian. Initial fixation was always at primary position, and the size and direction
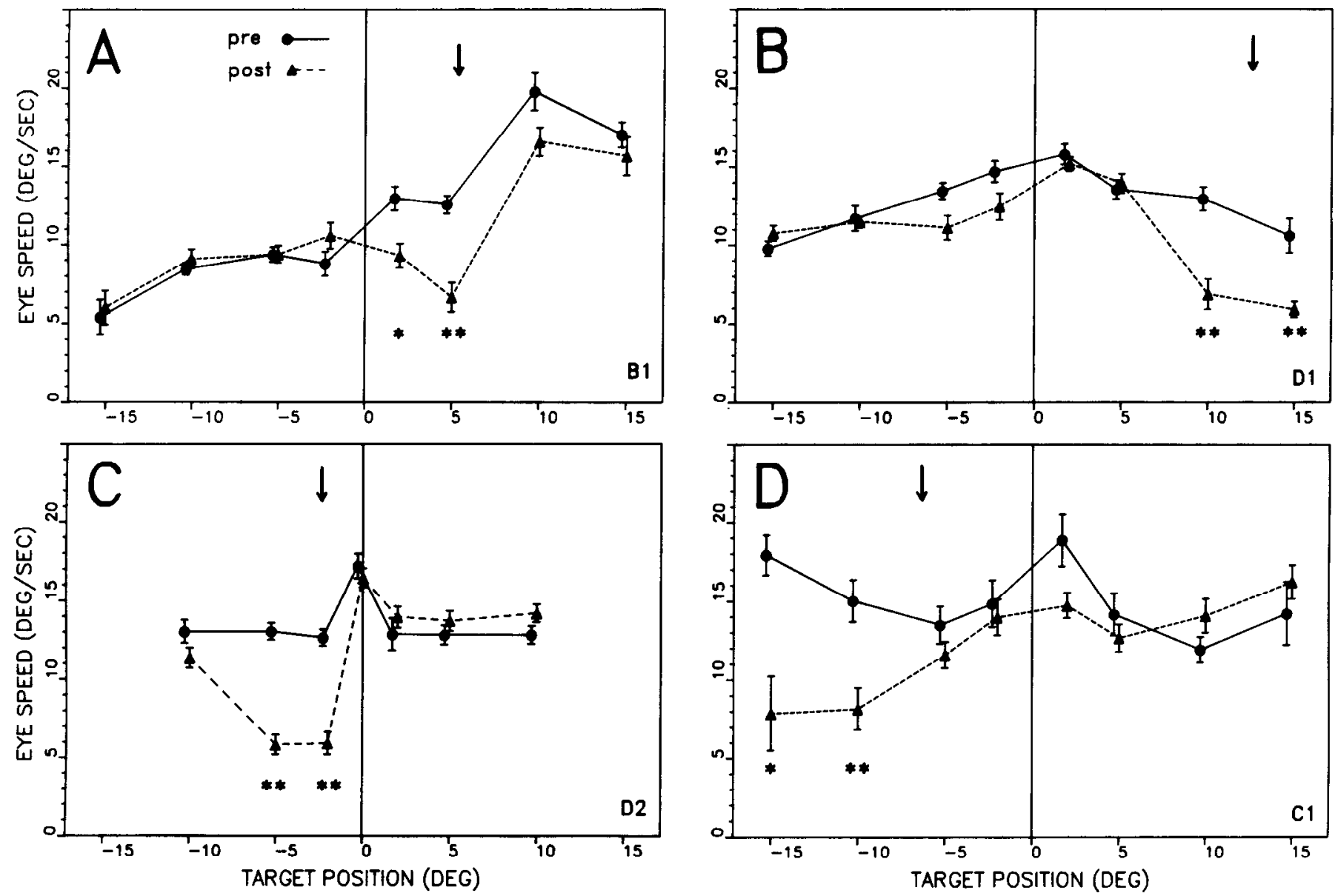

Figure 4. Spatial localization of the pursuit initiation deficit observed in each of the four experiments in this series. Step size from primary position is plotted on the abscissa (rightward, positive; leftward, negative). Horizontal eye speed, measured $70 \mathrm{msec}$ after the initial saccade in each trial, is plotted on the ordinate. The means and standard errors were calculated for 10 different pursuit responses obtained at each step location before and $24 \mathrm{hr}$ after each injection. The ramp portion of each trial was smooth horizontal motion away from primary position at $16 \% \mathrm{sec}$. The vertical arrow in each panel indicates the center of the MT receptive field recorded through the syringe needle at that injection site. In this and subsequent figures a double asterisk indicates a significance level of $p<0.001$, as determined by a $t$ test, and a single asterisk indicates $p<0.01$. $A$, Comparison of smooth pursuit eye speed before (solid line) and after (dashed line) the MT injection shows significant deficits $5^{\circ}$ and $2^{\circ}$ to the right of the center of gaze. The pronounced right-left asymmetry in pursuit eye speed was idiosyncratic for this monkey. $B$ to $D$. Pursuit initiation deficits in the remaining three experiments of this series. The impaired region of the visual field corresponds to the physiologically determined MT injection site in all cases. Throughout this paper we use the term "eye speed" rather than "eye velocity" since figures such as these include data from both rightward (positive target position) and leftward (negative target position) eye movements. 

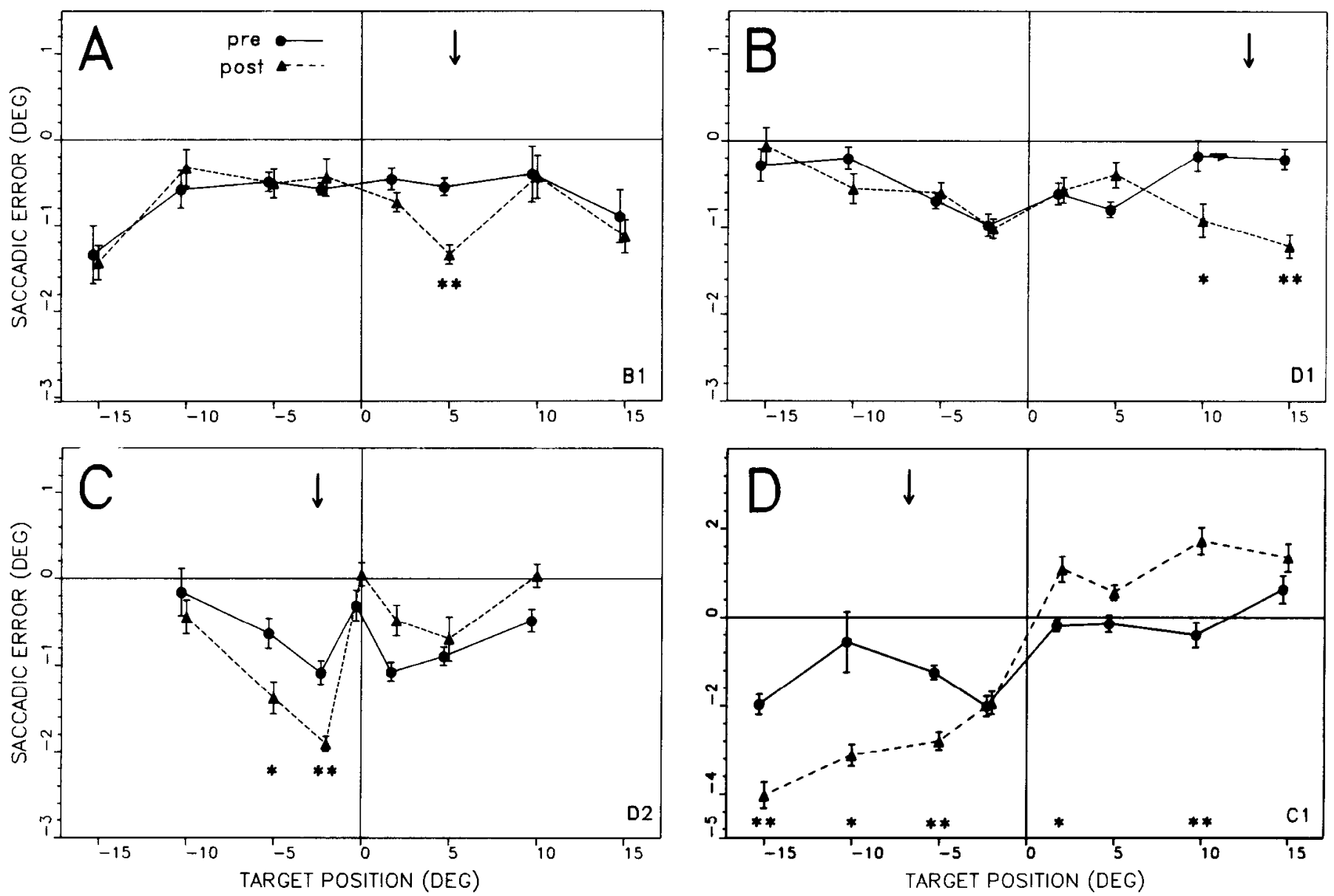

Figure 5. Deficits in saccades to moving targets following an ibotenic acid lesion of MT in each of the four experiments in this series. Saccadic error, defined as the difference between eye position and target position at the end of the initial saccade, is plotted on the ordinate. Amplitude and direction of the target step (which determine the retinal locus stimulated by subsequent target motion) are plotted on the abscissa. The sign of the saccadic error indicates whether the monkey was overshooting $(+)$ or undershooting $(-)$ the moving target. The means and standard errors were plotted for 10 different responses obtained at each step location before and $24 \mathrm{hr}$ after each injection. As in Figure 4 , the ramp portion of each trial was smooth horizontal motion away from primary position at $16 \% \mathrm{sec}$. $A$. The negative saccadic errors in the prelesion data show that, even for normal behavior, this monkey's initial saccades fell somewhat short of the moving target. After the MT injection, however, a significantly more pronounced error ( $t$ test, $p<0.001 ; * *)$ was present at the visual field location corresponding to the MT injection site (vertical arrow). $B$ to $D$. Saccadic error deficits for the remaining experiments in this series. Spatial location of the deficits corresponded to the physiologically determined sites of the MT injections. Labeling conventions are the same as for Figure 4.

of the target "step" with respect to primary position are plotted on the abscissa. For all of the trials in Figure 4, the direction of target motion after the step was away from primary position. A comparison of pre- and postlesion data in Figure $4 A$ reveals a clear reduction in postsaccadic eye speed for motion $5^{\circ}$ eccentric in the right hemifield ( $t$ test, $p<0.001$, Fig. 4A, double asterisk) and a smaller effect at $2^{\circ}$ eccentric ( $t$ test, $p<0.01$, Fig. $4 A$, single asterisk). No significant effect was observed for other locations in either hemifield. The multineuron receptive field which we recorded at the MT injection site was $6^{\circ}$ in diameter and centered $6^{\circ}$ to the right of the center of gaze on the horizontal meridian (Fig. $4 A$, arrow). The behavioral deficit, therefore, occurred at the expected location in the visual field, whereas control locations were unaffected. The pronounced right-left asymmetry in pursuit speed was idiosyncratic for this monkey and was not observed for the other animals in this series (Fig. $4, B$ to $D$ ).

Figure $4, B$ to $D$, illustrates the deficits in speed matching observed following injections in the other three hemispheres of this series. Each arrow indicates the center of the multineuron receptive field recorded at each MT injection site. Deficits in postlesion performance are clearly associated with each injection site. The deficient region of the visual field usually extends further into the peripheral as opposed to the central visual field with respect to the injection site.
This effect is probably due to the fact that the cortical magnification factor is larger in central than in peripheral MT (Gattass and Gross, 1981; Van Essen et al., 1981) and that our syringes approached MT at an angle such that any backflow of solution up the syringe needle would result in more peripheral portions of the visual field representation in MT being exposed to the ibotenic acid. The small fluctuations in performance that occurred at control locations were probably dependent on the monkey's motivational state and the degree of difficulty of each trial type for the monkey. However, the only locations for which the differences were statistically significant $(\rho<$ 0.01 or 0.001 ) were those indicated by the asterisks (Fig. $4, B$ to D).

Saccadic error. A second behavioral effect of the ibotenic acid injection can be seen in Figure $3 A$. In the prelesion traces, the saccadic eye movements made by the monkey in response to the displacement of the target were larger than the initial $5^{\circ}$ step of the target. The monkey therefore made allowance for the subsequent motion of the target when computing the amplitude of his initial saccade. Following the MT injection (Fig. 3A, lower traces), saccade amplitude was smaller than before, suggesting that MT lesions also reduced the monkey's ability to utilize motion information in programming such adjustments of saccade amplitude.

To measure this deficit we defined a quantity, saccadic error, as 

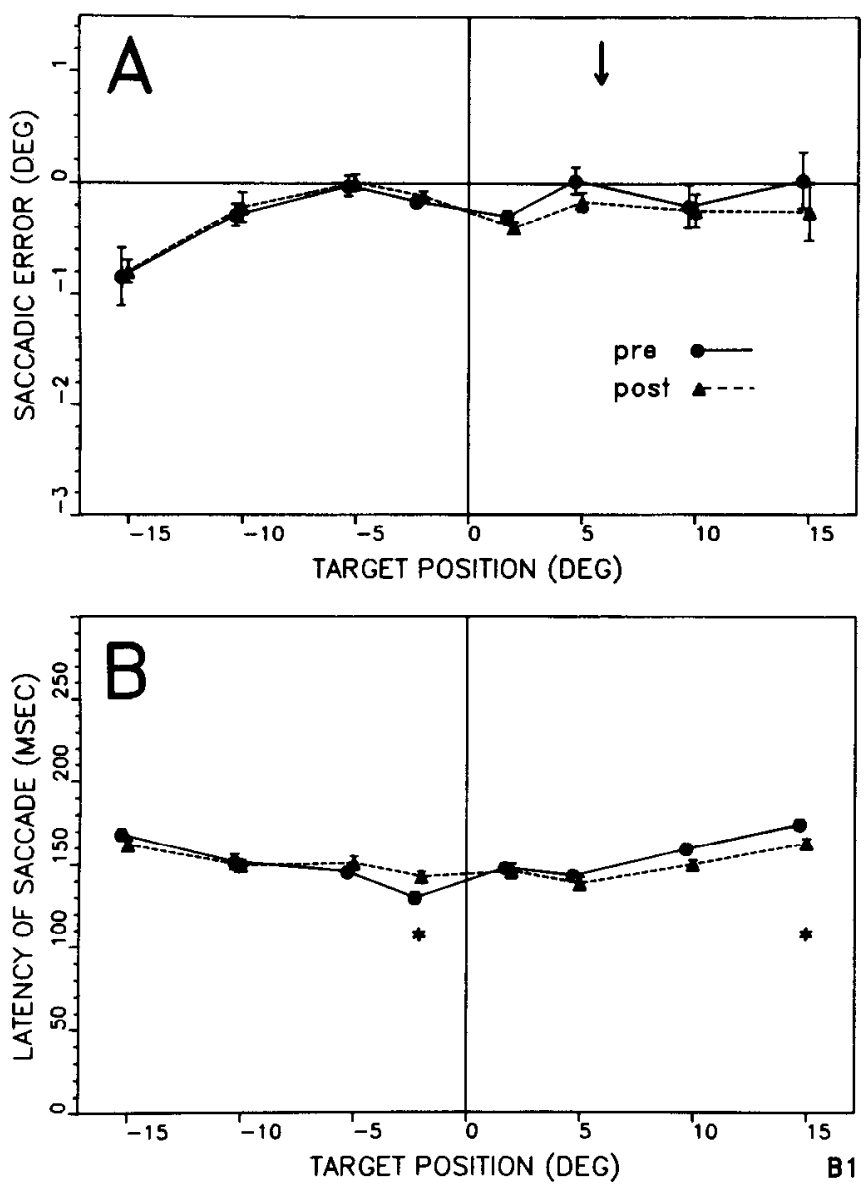

Figure 6. Lack of deficits when saccades were made to stationary targets. $A$. Saccadic error is shown as a function of step location in the visual field but the target remained stationary after the step. No deficits were observed even for the visual field location in which saccades to moving targets were impaired (see Fig. 5). Postlesion data were recorded at the same time as those in Figure $5 A$, and the display format is the same as in Figure $5 A . B$, Latencies of the initial saccade for the trials in $A$ are shown as a function of step location. Small but significant differences were observed at $-2^{\circ}$ and $+15^{\circ}$ which resulted from variations in behavior unrelated, presumably, to the MT injection. Performance was normal at $+5^{\circ}$, the location affected by the MT lesion. All labeling conventions are the same as in Figures 4 and 5. We observed no increase in latency of saccades to moving targets (see Figure $3 A$ )

the difference between target position and eye position at the end of the saccade. Eye position at the end of the saccade was measured from the eye traces recorded during the experiment, and target position was computed from the known step size and velocity of target motion. Figure $5 \mathrm{~A}$ shows saccadic error as a function of location in the visual field following this injection. Once again, the mean and standard error of 10 trials at each location are shown before and $24 \mathrm{hr}$ after the lesion. Postlesion performance closely matched prelesion performance at all locations in the visual field except for the point $5^{\circ}$ to the right - the center of the region affected by the MT lesion. Here the saccade amplitude was significantly smaller ( $t$ test, $p<0.001$ ) after the lesion. Whereas initial saccades at this point fell about $0.5^{\circ}$ short of the moving target before the lesion, the saccades fell, on the average, $1.4^{\circ}$ short of the moving target after the lesion. This deficit is small in absolute terms but is substantial when considered as a fraction of the total distance the target moved in the interval between its appearance and the end of the saccade; in this $200-\mathrm{msec}$ interval the target moved $3.2^{\circ}$, yet the average saccadic error was $1.4^{\circ}$. Following the lesion, the monkey allowed for roughly $50 \%$ of the distance traveled by the target, whereas he allowed for about $85 \%$ before the lesion.
Saccadic error deficits following the other three injections are illustrated in Figure $5, B$ to $D$. The result in Figure $5 B$ was particularly clear: the deficits at $+10^{\circ}$ and $+15^{\circ}$ were centered on the receptive field recorded at the injection site (Fig. 5B, arrow) and were the same locations for which speed matching deficits were observed (Fig. 4B). Saccadic error deficits were present in Figure 5, $C$ and $D$ but were accompanied by changes in saccade amplitude of the opposite polarity in the ipsilateral hemifield. It is possible that these changes in the ipsilateral hemifield reflect a general increase in effort as the monkeys tried to compensate for deficient performance in the damaged hemifield.

To control for the possibility that the motion-related deficits simply resulted from a general deterioration in the monkey's ability to localize visual stimuli in the damaged portion of the visual field, we measured saccadic error in the "step" trials in which the monkey made saccades to stationary targets. The data for injection B1 (Figs. $3,4 A$, and $5 A$ ) are displayed in Figure $6 A$ in the same form as the data of Figure 5. Postlesion performance is similar to prelesion performance at all points in the visual field, including the location that was deficient when saccades were made to moving targets. As a further check of the monkey's performance on this control, the latencies for saccades to the stationary targets are shown in Figure $6 B$. Small (10 to $12 \mathrm{msec}$ ) but significant differences were observed at $-5^{\circ}$ and $+15^{\circ}$, but no differences were observed in the region affected by the MT lesion. Both amplitude and latency of saccades to stationary targets seemed unaffected following each injection. Thus, MT lesions reduce a monkey's ability to utilize motion information for the adjustment of saccade amplitude and for the initiation of appropriate pursuit speeds, while leaving intact his ability to utilize static position information.

In one monkey, we made an injection of normal saline through the same guide tube used for a subsequent injection of ibotenic acid (Table I). No deficit was present following the saline injection, but both deficits were observed following the subsequent ibotenic acid injection into the same location.

Tracking toward primary position. All of the pursuit data presented until now have been for tracking-away trials in which the target "stepped" to some point on the horizontal meridian and moved away from primary position. The speed matching and saccadic error deficits were also observed for tracking-back trials in which the target moved toward primary position after the step. Figure 7 shows data for tracking-back trials from injection D2. In Figure $7 A$, the target stepped $10^{\circ}$ to the monkey's left and returned toward primary position at $16^{\circ} / \mathrm{sec}$. The speed matching deficit in the initial pursuit interval is clearly seen in the composite eye speed records in Figure $7 B$. Initial eye speed was again reduced by about half and did not reach prelesion levels until roughly $125 \mathrm{msec}$ following the initial saccade. Saccadic error in the prelesion trials was virtually zero as the monkey adjusted the amplitude of the initial saccade to allow for the motion of the target. In the postlesion trials, however, there was an average saccadic error of $1^{\circ}$ as the monkey overshot the moving target with his initial saccade.

Rashbass (1961) first pointed out that, for certain "step" sizes in tracking-back trials, smooth pursuit can be initiated and maintained without the added presence of a saccadic eye movement. An example of such a case is shown in the prelesion traces in Figure $7 \mathrm{C}$. The target was displaced $2^{\circ}$ to the monkey's left, and the return motion was such that pursuit was initiated in a relatively "pure" form without the influence of a saccade. For our purposes this feature complicated the analysis, since the initial saccade was an important feature of the measurement of both behavioral deficits. We therefore neglected such trials in our quantitative analysis of tracking-back data (in Fig. 8, for example), but Figure 7,C and $D$, shows qualitatively that the motion-related deficits were present in such trials when the target motion occurred in the damaged portion of the visual field. Although no saccade interrupted pursuit initiation in the prelesion trials, a small saccade did occur in the postlesion trials which caused the monkey to overshoot the moving target. As in the previously 

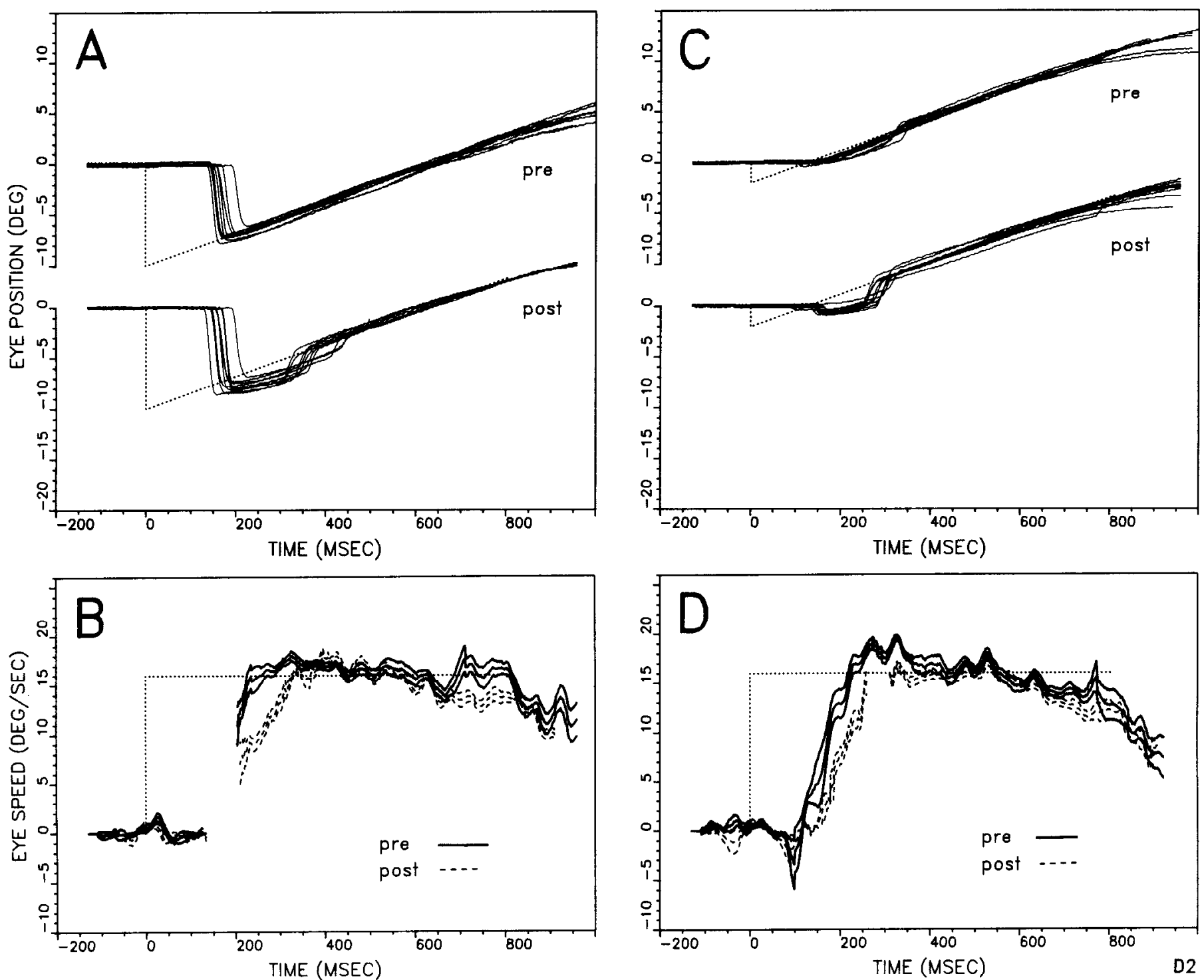

Figure 7. Deficits in pursuit initiation for trials in which target motion was toward primary position following the step. A, Target position (dashed line) and eye position ( 10 superimposed traces, solid lines) are plotted as a function of time. The target stepped $10^{\circ}$ to the monkey's left and returned at $16^{\circ} / \mathrm{sec}$ toward primary position. The initial saccades in the prelesion traces were of an appropriate amplitude so that the eyes were directed precisely toward the moving target. In the postlesion traces recorded $24 \mathrm{hr}$ after injection (centered $2^{\circ}$ to the left of primary position), the initial saccades consistently resulted in an overshoot of the moving target. $B$. Mean smooth eye speed (with standard errors) is plotted as a function of time for the pre- and postlesion traces illustrated in $A$. Smooth pursuit eye speed was lower in the postlesion trials for about $100 \mathrm{msec}$ following the initial saccade. $C$, The target step $\left(-2^{\circ}\right)$ and motion ( $16 \% \mathrm{sec}$ toward primary position) were arranged so that smooth pursuit was initiated in the prelesion traces without interruption by saccades. In the postlesion traces recorded $24 \mathrm{hr}$ after the same MT injection as in $A$, the monkey made a small saccade in response to the same stimulus which resulted in an overshoot of the moving target by the eye. $D$. Mean smooth eye speed (with standard errors) is shown for the pre-and postlesion traces illustrated in C. Postlesion eye speed lags prelesion performance for the first 100 to $200 \mathrm{msec}$ of the response. Display formats and all labeling conventions are the same as those in Figure 3.

presented cases, the monkey failed to take full account of the motion of the target in executing a saccade, although in this case the error lay more in the decision to make a saccade than in the precise amplitude of the saccade. Composite eye speed for these trials is plotted in Figure $7 D$, and postlesion eye speed lagged prelesion performance for the first 100 to $200 \mathrm{msec}$ of pursuit.

Quantitative data for tracking-back trials from injection D2 are shown in Figure 8. Eye speed measured $70 \mathrm{msec}$ after the saccade is plotted against target position in Figure $8 A$, and saccadic error is plotted in Figure $8 B$. Both measures show deficits at $-5^{\circ}$ and $-10^{\circ}$, although the speed matching deficit falls just short of statistical significance at $-5^{\circ}$. The fact that saccadic error is positive in this case rather than negative as in Figure 5 indicates that the error was an overshoot rather than an undershoot. It is interesting to note that, for this injection (D2), the monkey had deficits for tracking-back trials at $-10^{\circ}$ but was normal for tracking-away trials at $-10^{\circ}$ (Fig. $4 \mathrm{C}$ ). This is understandable since the actual retinal locus stimulated by the target motion was $-10^{\circ}$ to $-8^{\circ}$ for tracking-back trials and $-10^{\circ}$ to $-12^{\circ}$ for tracking-away trials. The boundary of the deficient patch of the visual hemifield was apparently very close to $-10^{\circ}$.

Presaccadic initiation of pursuit. In recent work, Lisberger and Westbrook (1985) have exploited the short latency of the pursuit system relative to the saccadic system to study the pursuit response to motion at various retinal locations before the occurrence of the initial saccade. Ths presaccadic pursuit response is most reliably obtained under experimental conditions in which the pursuit latency is minimized and the saccade latency is maximized. We employed such conditions for injection C1 to examine the effect of MT lesions on pursuit initiation without an intervening saccade. In these experiments the target light came on at the same time that the fixation 

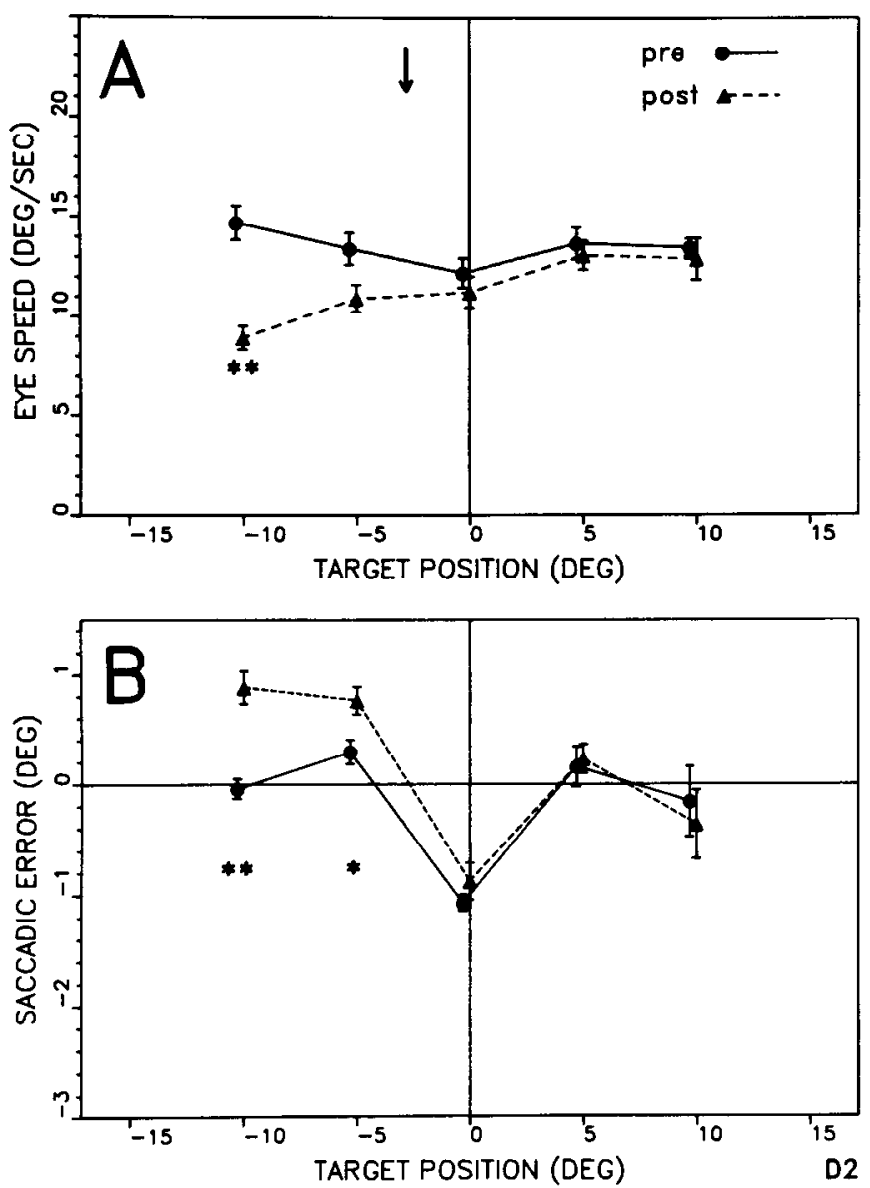

Figure 8. Spatial localization of speed matching and saccadic error deficits in tracking-back trials in experiment D2 (same lesion shown in Figure 7). A, Smooth pursuit eye speed, measured $70 \mathrm{msec}$ following the end of the initial saccade, is shown as a function of step location (target position). The deficit at $-5^{\circ}(\rho<0.02)$ fell just short of our standards of statistical significance. $B$, Saccadic error is plotted as a function of step location. The positive values of the postlesion data indicate an overshoot of the moving target by the initial saccade. Display format and labeling conventions are the same as in Figures 4 and 5 .

light came on, but the target did not move until the fixation light went out. Data from a location in the visual field affected by the MT lesion are shown in Figure 9 . In the prelesion traces of Figure $9 A$, one can see a small but regular interval of pursuit which preceded the initial saccade in each trial (Fig. 9A, arrow). This pursuit response is directly attributable to motion on the retina at an eccentricity determined by the size of the target "step," since the eyes had not yet been moved significantly by a saccade. In the postlesion traces the presaccadic pursuit was abolished. This result can be seen more clearly in the composite eye speed traces in Figure 9B. Prelesion, the presaccadic pursuit reached an eye speed of $6 \% \mathrm{sec}$ before being interrupted by the initial saccade. In the postlesion data, mean eye speed did not depart from zero before the saccade. This pursuit deficit that occurs before a saccade to a moving target is therefore consistent with the speed matching deficit in the initial interval following the saccade.

Recovery. Each monkey recovered from its deficits during the first week after the injection. The time course of recovery was such that the best data were invariably obtained within the first $24 \mathrm{hr}$ following the injection, and all aspects of the response-speed matching, saccadic error, and presaccadic initiation-were statistically indistinguishable from prelesion standards within days following the lesion. Figure 10 documents the recovery for speed matching in injection D2. The prelesion and 24-hr postlesion eye speeds are the same as those shown in Figure $4 C$, but the speeds illustrated by the diamonds and dotted line were recorded on the 8th day postlesion. The monkey had performed the tasks on 5 of the 8 postlesion days. The monkey recovered normal function at the deficient locations while maintaining relatively stable performance at control locations.

Several caveats should be kept in mind since we have not yet studied the recovery process in detail. First, the deficits we observed seem attributable to a difficulty on the monkey's part in gauging the speed of a moving target (see "Discussion"). Since the speed of our target was always $16^{\circ} / \mathrm{sec}$, a major predictive element was present in our experiments which may have abetted the recovery process. Recovery may be more prolonged when the predictive element is removed. Second, occasional trials seemed to indicate that the monkey had a behavioral deficit even after the aggregate behavior was statistically indistinguishable from prelesion behavior. Such trials may have increased in frequency as the monkeys became tired or satiated. Therefore, we are hesitant to assert that a monkey was operating at prelesion efficiency even after the quantitative evaluation indicated that recovery was complete. Third, we have not yet evaluated the effect of visual experience or practice on the rate of recovery. The deficits may be more long lasting in the absence of perceptual feedback concerning the system's deficient state.

Histological reconstruction. Cortical damage resulting from an injection of ibotenic acid is easily observed in histological sections stained for cell bodies. A parasagittal section illustrating the damage to MT following injection C1 is shown in Figure 11A. The lesion is localized to MT on the posterior bank of the STS and its borders are sharp, as though they indicate a specific isoconcentration line for lethality. Figure $11 B$ provides a higher power view of the lesion boundary in the same section. Cortex to the right of the boundary appears normal, with its typical laminar structure and columnar stacking of cell bodies in the upper layers. Cortex to the left is grossly damaged, with a dearth of neurons and a massive gliosis being its most striking characteristics. Apparently vital neurons can be observed occasionally in the damaged region (Fig. 11D), but we estimate that the survival rate is under $5 \%$. To the extent that surviving neurons receive input from nearby cortical interneurons, their inputs and functional properties must be substantially altered following the lesions. Figure $11 \mathrm{C}$ shows, in the same view as Figure $11 B$, a nearby section stained for myelinated fibers. As in all of the sections we examined, the white matter appears to stain normally, reinforcing the notion that ibotenic acid spares fibers of passage. Terminal fibers in the cortex, however, appear poorly stained and discontinuous. We cannot tell whether this abnormal staining reflects primary damage to terminal fibers by the ibotenic acid, or whether it is secondary to the destruction of the targets of the fibers or to a general deterioration of the cortical tissue.

The full extent of cortical damage in each hemisphere was plotted on outlines of histological sections and transferred to a two-dimensional cortical map created from the section outlines by the method of Van Essen and Maunsell (1980). Each map represents the cortex of a particular hemisphere after an "unfolding" process so that relevant information can be examined without the complications of the naturally occurring sulci. Figure 12 contains such a reconstruction for each of the four hemispheres in this study. For ease of comparison, maps from right hemispheres were mirror-reversed so that all maps appear as left hemispheres. Each map represents a similar region of cortex so that the ibotenic acid-induced damage can be seen in relation to the myeloarchitectonic borders of MT and compared across injections. The region contained in the map includes the lateral two-thirds of the lunate sulcus and the posteromedial half of the STS as indicated in the inset in Figure 12A. The fundus of each sulcus is shown by the dashed lines, and the borders of the sulci are indicated by the heavy solid lines in Figure 12A. The orientation of the two sulci in the maps is similar to their orientation in the inset except that they are "unfolded" so that cortex within the sulci may be examined. Thus, the posterior bank of each sulcus lies to the left of its fundus and the anterior bank to the right. MT is indicated on the posterior bank of the STS in each map, and 

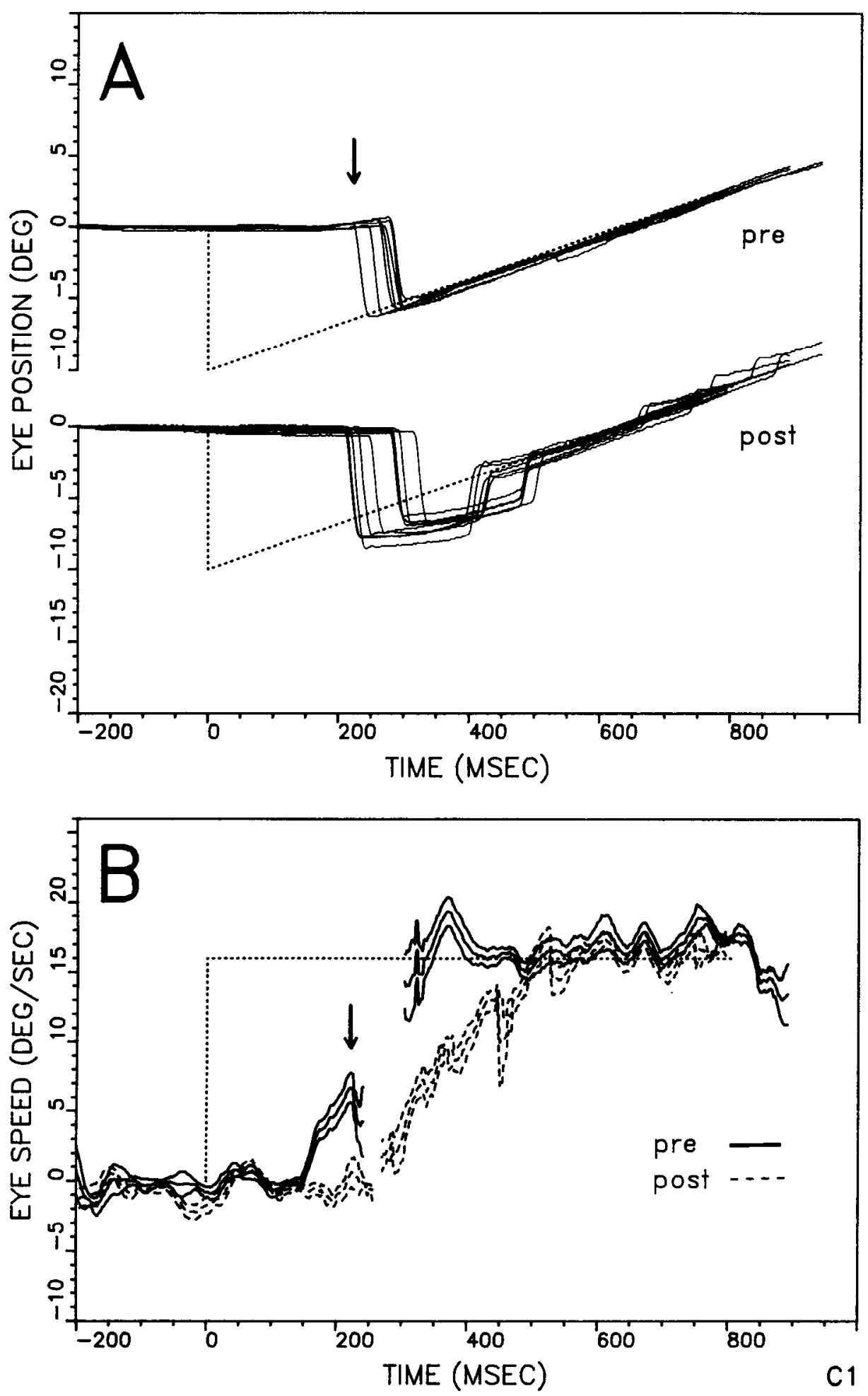

Figure 9. Elimination of presaccadic pursuit by an ibotenic acid injection in MT. $A$, In this experiment a brief interval of smooth pursuit preceded the initial saccade in the prelesion traces (arrow). This response can be unambiguously attributed to visual information derived from the peripheral retina $\left(-10^{\circ}\right.$ to $\left.-7^{\circ}\right)$, since a saccade had not yet brought the fovea near the target. In the postlesion traces recorded 24 hr after the injection, the presaccadic pursuit response was completely eliminated. $B$, Mean smooth eye speed (with standard errors) is shown as a function of time for the pre- and postlesion traces in A. The elimination of the presaccadic pursuit response is particularly clear in this panel. Mean smooth eye speed before the initial saccade reached an average of $7 \% \mathrm{sec}$ in the prelesion trials (arrow) but did not depart from base line in the postlesion trials. The overshoot of the moving target due to saccadic error can be seen qualitatively in $A$, and the deficit in smooth eye speed after the initial saccade is present in $B$. Display format and labeling conventions are the same as in Figure 3 . boundaries of the damaged cortex are shown as well. Damage in the STS was restricted almost completely to MT except in the case of injection B1 (Fig. 12B). The central core of damage to all cortical layers (solid black region) varied in size among the cases, from a high of $2.5 \times 2 \mathrm{~mm}$ for injection C1 (Fig. $12 A$ ) to a low of $1 \times 1 \mathrm{~mm}$ for $\mathrm{D} 1$ and $\mathrm{D} 2$ (Fig. 12, $C$ and $D$ ). The lesion in $\mathrm{B} 1$ was actually due to three sequential, closely spaced injections. Only the first injection is considered in this paper. The lesions in C1, D1, and D2 each resulted from a single $1-\mu$ injection. The size of the visual field deficit could not be correlated with the extent of tissue damage, in part because the peripheral boundary of the deficient region of the visual field was not identified following injections $C 1$ and D1.
The primary problem we encountered in our attempts to make specific MT lesions was damage on the anterior bank of the lunate sulcus, which resulted from back diffusion of ibotenic acid along the syringe needle as for injection D2. Injection C1 escaped this contamination entirely, and the problem was minor for injection D1. In B1 the relevant injection was the most lateral in the series of three into $M T$, and the most lateral penetration resulted in minimal damage in the lunate in Figure 12B. Therefore, we strongly suspect that injection D2 is the only injection for which our effort may have been compromised by damage in the lunate sulcus. This possibility is substantiated by small but significant changes in the saccades to stationary 


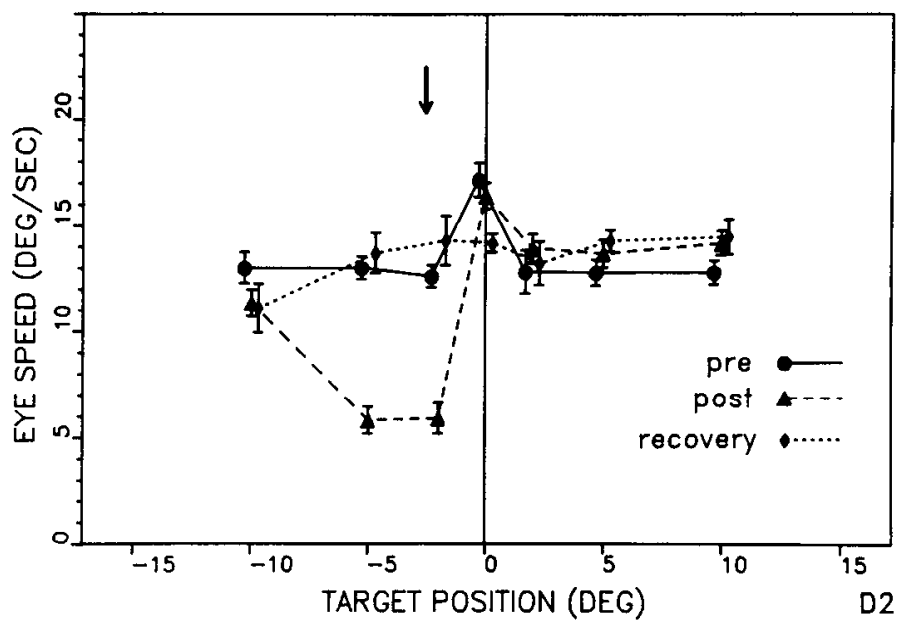

Figure 10. Recovery of function following ibotenic acid lesions of MT. Shown is the smooth eye speed measured $70 \mathrm{msec}$ after the initial saccade for prelesion (solid line), $24 \mathrm{hr}$ postlesion (dashed line), and 8 days postlesion (dotted line). After 8 days, the monkey had recovered sufficiently so that its behavior was statistically indistinguishable from prelesion performance at the previously impaired locations in the visual field. The difference between pre and recovery at $0^{\circ}$ was statistically significant $(p<0.001)$ but was presumably unrelated to the lesion since no deficit was present at $0^{\circ}$ on the first day postlesion. Display format and labeling conventions are the same as those in Figure 4, and the pre and 24-hr pattern data are the same as those illustrated in Figure $4 C$.

targets which we observed for stimuli presented at $-5^{\circ}$ on the horizontal meridian following this injection. However, visual field deficits due to the MT damage were observed from $-2^{\circ}$ to $-10^{\circ}$ following this injection (Figs. $4 \mathrm{C}$ and 8 ), and saccades to stationary stimuli at $-2^{\circ}$ and $-10^{\circ}$ were normal. In sum, the lesions appear to have been restricted primarily to MT, and the histological results therefore support the view that the observed behavioral deficits reflect disruptions in the functional integrity of MT.

An unusual pathology. In one of our animals ( $D$; see Table I) we observed an unusual cortical pathology which is illustrated in Figure 13. The pathology was a series of small microlesions in layer IV of an extended region of dorsal extrastriate cortex. It is apparent in the low power micrographs of Figure 13, $A$ and $B$, that the microlesions occurred periodically in the affected cortex with an average interlesion distance of 500 to $600 \mu \mathrm{m}$. The lesions probably occurred as "bands" rather than "blobs," since they appeared elongated in one dimension in tangentially sectioned regions of cortex. The myelinrich zones in Figure $13 B$ superimpose directly upon the microlesions in Figure $13 A$ and presumably reflect the same underlying pathology. The high power micrographs in Figure $13, C$ and $D$, illustrate the appearance of a single microlesion in Nisst- and myelin-stained sections, respectively. The lesion clearly results from cell loss in the upper two-thirds of layer $\mathrm{N}$ and perhaps in the lowest extent of layer III as woll. Examination of the myclin-staincd scction in Figure 13D reveals that the dark staining zones reflect a proliferation of fibers in the area of cell loss rather than an increase in nonspecific background stain. Ibotenic acid injections were made in both hemispheres in this animal, and the pathology was present bilaterally. In the hemisphere with the least extensive damage, the lesions affected buried cortex near the confluence of the lunate $(L S)$, intraparietal (IPS), and parieto-occipital (POS) sulci, including the annectant gyrus $(A G)$ and nearby walls of all three sulci. In the more extensively damaged hemisphere, microlesions occurred in the abovementioned locations and in a large region of the STS as well. Light damage was also observed in the lateral extent of the inferior occipital sulcus and in a small portion of the central sulcus. Striate cortex was not affected in either hemisphere. Cortex affected by the microlesions included the MT injection site in the latter hemisphere, but not in the former. Curiously, microlesions were never observed on the surface of the cortex, only in sulci.
We are currently unable to tell whether the microlesions resulted from the presence of ibotenic acid in the cortex or to some pathological process unrelated to our study, and we present these observations primarily as a precaution concerning possible complications of our technique. The pathology probably reflects some underlying organizational feature of the cortex, but no known pattern of anatomical connections appears to match the pattern of microlesions. Whatever the cause of the pathology, there were no aspects of this monkey's behavior that differed from the other monkeys in this study, either before or after the lesion; thus, we feel confident that the pathology had no bearing on the results of this study.

\section{Discussion}

Nature of the behavioral deficits. The results of these experiments provide the first behavioral evidence that the middle temporal visual area of the monkey is selectively involved in the analysis of visual motion. We observed two deficits following MT lesions: an impaired ability to match smooth pursuit eye speed to target speed, and an impaired ability to compensate for target motion when making saccades to moving stimuli. Both deficits appeared to reflect a common deficiency in the processing of motion by the visual system rather than deficiencies in the oculomotor control systems. We will summarize the evidence for this interpretation.

First, the deficits were consistently related to a specific portion of the visual field as opposed to a specific aspect of oculomotor performance. This observation is particularly telling since deficits appeared for two types of oculomotor response-smooth pursuit eye movements and saccadic eye movements-which are thought to be controlled by different neural subsystems (Robinson, 1981). In addition, individual pursuit responses contained a period of deficient tracking while visual input was received from the deficient portion of the visual field, but they rapidly returned to normal when an intervening saccade brought the visual stimulus to an intact portion of the visual field. The deficient portion of the visual field was predictable from the physiologically recorded receptive fields at the site of the ibotenic acid injection in MT, and single neuron recording experiments have failed to show any oculomotor influence on the responses of MT neurons (Newsome and Wurtz, 1981; Wurtz et al. 1984b).

Second, the deficits were observed only when eye movements were made in response to moving visual targets. Control trials generally showed that the monkey's saccades to stationary targets were normal in amplitude and latency even when the targets appeared in visual field locations where the responses to moving stimuli were deficient. Although this control obviously does not constitute a thorough appraisal of a monkey's capacity for visualizing stationary stimuli, the result suggests that the effects of MT lesions are more severe for moving than non-moving stimuli and thus support the physiologically based hypothesis that MT is selectively involved in the analysis of visual motion.

Third, both deficits appear to reflect an underestimation by the monkey of the speed of moving visual stimuli. The monkey is not totally oblivious of moving stimuli in the deficient portion of the visual field: it continues to generate a smooth pursuit response to the moving stimulus and continues to compensate somewhat for the motion of the target in programming saccade amplitude. However, the pursuit response consistently results in an eye spced that is slower than target speed, and the pattern of saccadic error also reveals an underestimation of target speed: if motion of the target is away from primary position, monkeys consistently undershoot the target with a saccade; if motion is toward primary position, monkeys consistently overshoot with a saccade. This result was somewhat ironic since the cardinal characteristic of MT neurons has been their direction selectivity, speed selectivity being only recently studied (Baker et al., 1981; Maunsell and Van Essen, 1983a). Although we observed no deficits in the monkeys' judgment of direction, we required a relatively crude direction judgment of the monkeys ("toward" or "away from" primary position), and we did not analyze 

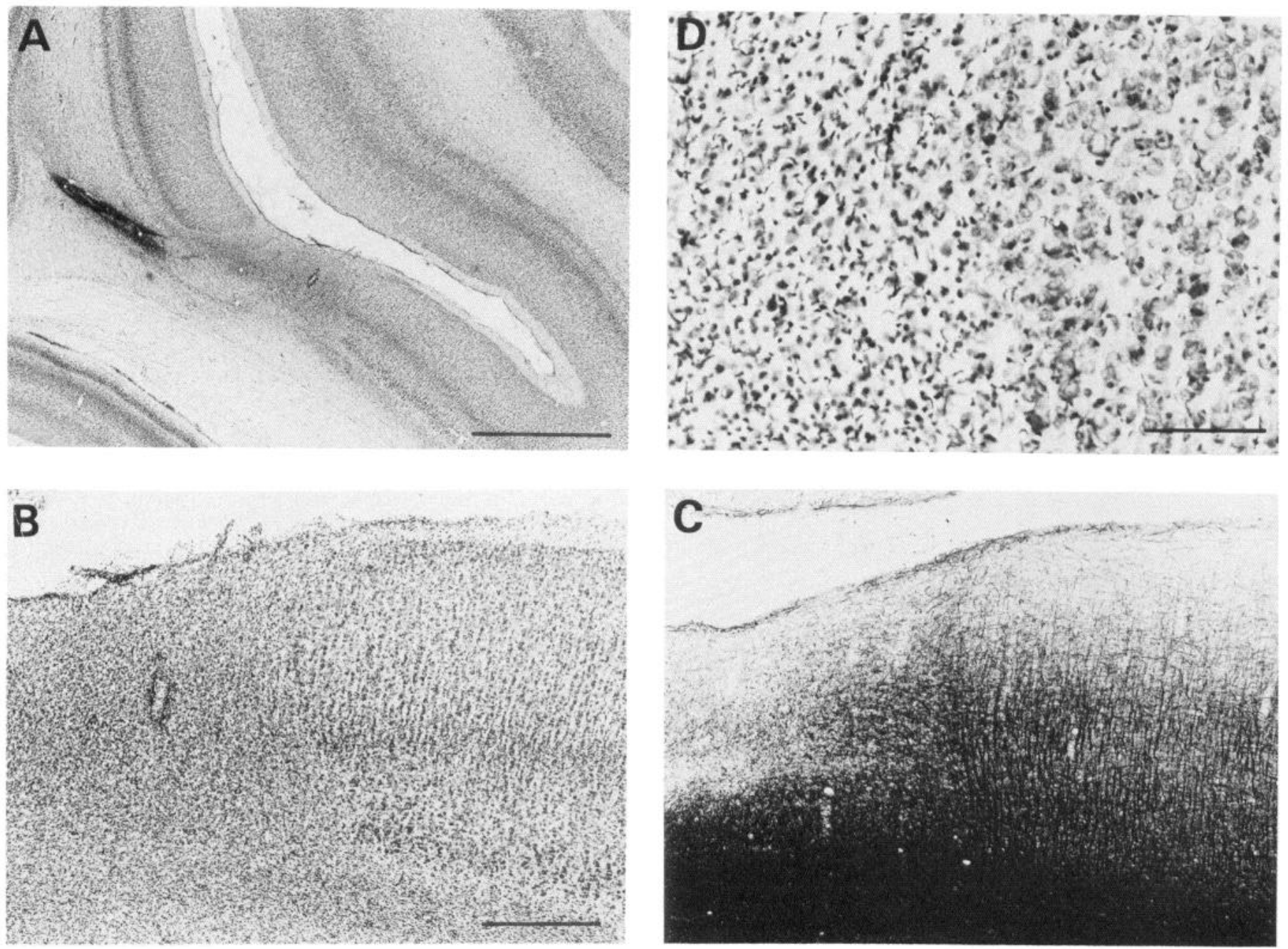

Figure 11. Histological effects of an ibotenic acid injection into MT in experiment C1. A, A parasagittal section stained with cresyl violet for cell bodies showing the ventral portion of the superior temporal sulcus. Dorsal is upward and anterior is to the right. The boundaries of the damaged area are easily visible and are restricted to the posteroventral bank of the sulcus in MT. Damage caused by passage of the Hamilton syringe needle is visible in the white matter underneath MT. A small portion of striate cortex in the calcarine sulcus is at the lower left, and a small portion of the sylvian sulcus is at the upper right. The calibration bar is $2.0 \mathrm{~mm}$. B, A higher power micrograph of one of the lesion boundaries in $A$. The blood vessel in the damaged cortex is visible in both micrographs. Cortex to the right of the boundary exhibits the normal columnar stacking of cell bodies and laminar structure characteristic of this area. Cortex to the left of the boundary is grossly disrupted: there is a pronounced loss of neuronal cell bodies and a massive gliosis. Calibration bar, 500 $\mu \mathrm{m}$. C, A nearby section stained for myelinated fibers by the method of Gallyas (1979). The view and calibration are the same as in B. D, A high power photomicrograph of the lesion boundary in layers II and III. Calibration bar, $100 \mu \mathrm{m}$.

vertical eye movements. It will be interesting to see whether appropriately designed experiments will reveal deficits in the monkey's response to the direction as well as the speed of moving visual stimuli.

Other pursuit deficits following cortical lesions. There are two general classes of pursuit deficits observed after cortical lesions in primates that should be compared to the present results: lesions of visual areas preceding MT in the cortical visual pathway, and lesions of higher order cortical areas. MT receives direct inputs from striate cortex, from a number of extrastriate visual areas, and from the pulvinar as well. No data are yet available concerning the effects on pursuit of lesions of identified extrastriate areas other than MT. However, several studies of pursuit deficits following striate cortex lesions have recently been reported (Goldberg et al., 1982; Zee et al., 1982; Segraves et al., 1983). Goldberg et al. (1982) tested monkeys with a unilateral striate ablation on a variety of step-ramp tracking tasks similar to those employed in this study. They demonstrated conclusively that the profound deficits in pursuit initiation and saccades to moving targets which follow ablation of striate cortex are due to deficient visual input from the affected region of the visual field: pursuit initiation and saccades to moving stimuli in the intact hemifield were normal. In contrast to our experiments, the unilateral striate monkeys appeared to have a permanent (although not absolute) impairment in their ability to respond to visual motion. Similar results were obtained by Zee et al. (1982), following bilateral occipital lobectomies, although the bilateral animals experienced more recovery than did the unilateral cases reported by Goldberg et al. (1982). Thus, the deficits in eye movements to moving targets following striate lesions appear qualitatively similar to, although quantitatively more severe and long lasting than, those following MT lesions.

In contrast to the effects of MT lesions, however, the striate lesions had effects on saccades to stationary targets as well as moving targets. Saccades to stationary targets were initially abolished after the ablation of striate cortex (as reported previously by Mohler and Wurtz, 1977). Thus, striate lesions dramatically affect the visual guidance of eye movements to both stationary and moving stimuli, whereas the effects of MT lesions appear to be selective for motion. We do not believe that this difference in the effects of striate and MT lesions can be attributed to differences in the quality or completeness of the cortical lesions produced by ibotenic acid as opposed to surgical ablation. We have made small ibotenic acid 

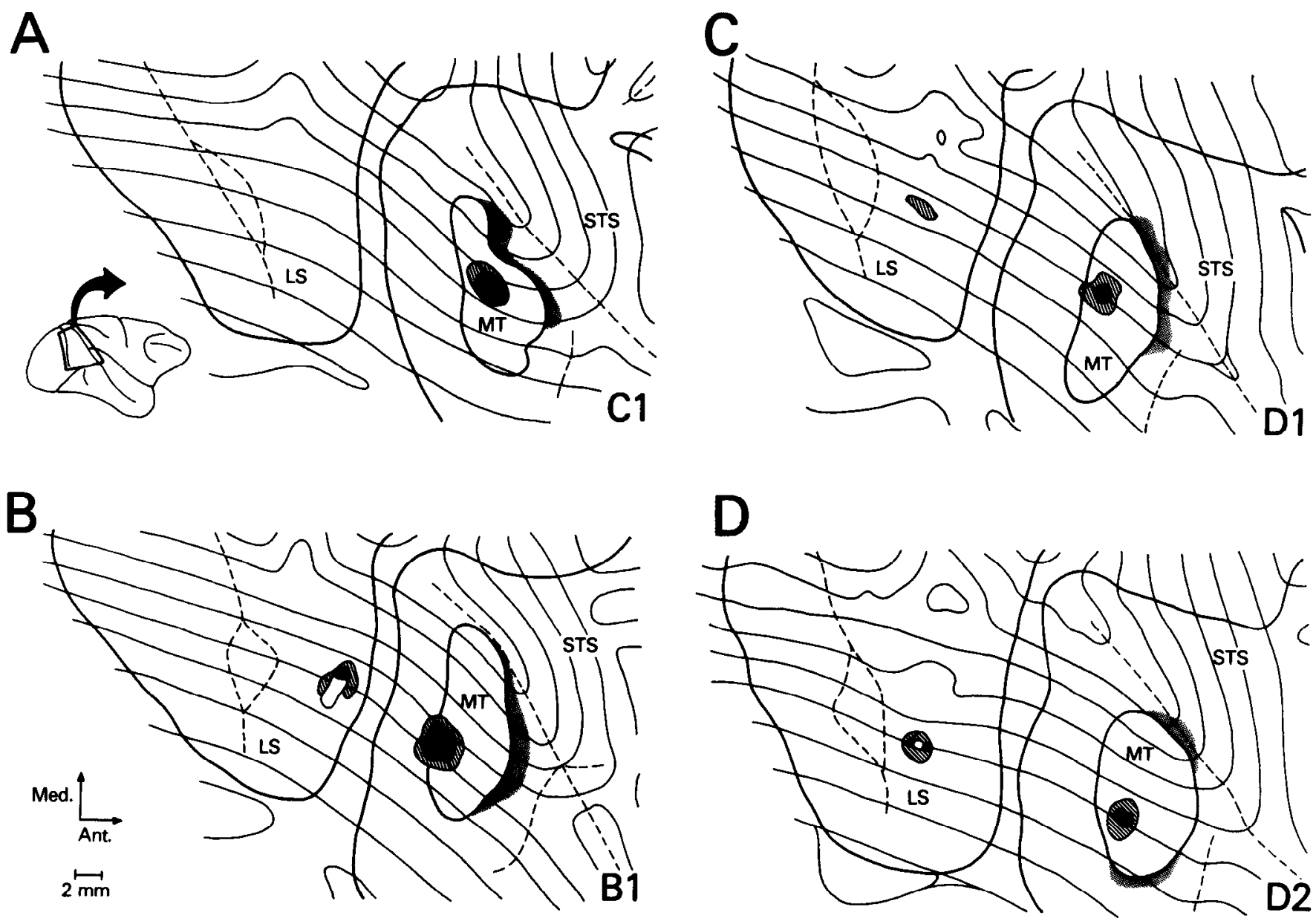

Figure 12. Two-dimensional reconstructions of the cortex created by the method of Van Essen and Maunsell (1980). Complete histological results for each of the four hemispheres in this study are shown in $A$ to $D$. The area of cortex reconstructed is similar for all four hemispheres and is shown in the inset in $A$. For ease of comparison, maps made from right hemispheres were mirror-reversed so that all four maps appear in the left hemisphere format. The light gauge solid lines are the "unfolded" layer IV contours from which the maps were made. The dashed lines indicate the fundi of the lunate (LS) and superior temporal sulci (STS), and the heavy solid lines show the boundaries of the sulci. The lunate sulcus is to the left (posterior) and the superior temporal sulcus is to the right. Medial is upward in the maps. The boundaries of MT were determined from myelin-stained sections and are also shown in heavy solid lines. The gray-toned area along one border of MT indicates a region of uncertainty in determining the border. Cortical damage created by the ibotenic acid is shown by the solid black and hatched areas. The solid black area indicates damage in all cortical layers, and the hatched areas indicate regions of partial laminar destruction. The white regions in the lunate sulcus surrounded (or nearly so) by ibotenic acid damage are scars left by the guide tubes. The guide tubes were present during pre- and postlesion data collection and should not have affected the results. The experiment name is given at the lower righthand corner of each map.

lesions of striate cortex, and postlesion deficits in the monkey's response to stationary as well as moving targets were as profound as those following ablation, although they were more transient (Newsome et al., 1985). Therefore, the fact that deficits following MT lesions were restricted to moving targets probably reflects the functional specialization of MT for the analysis of motion.

The effects of lesions of higher cortical areas on eye movements to moving targets have been most intensively studicd in humans. In general, clinically informative cases are characterized by damage to the parieto-occipital region of cortex and result in tracking deficits when target motion is toward the side of the lesion (for review, see Troost and Abel, 1982). These deficits appear to be very different from the deficits we observed following MT lesions in monkeys which were related to motion in a particular part of the visual field regardless of the direction of tracking. In humans, deficits were observed for a particular direction of tracking and thus appear to be related to some aspect of motor performance. The human subjects were generally studied during steady foveal tracking of sinusoidally moving targets. Since precise control over the location of the target on the retina is not achieved in this situation, it is difficult to know with certainty whether the behavioral deficits are truly related to a particular motor response (direction of tracking) or to a particular visual hemifield.

A recent study of oculomotor functions in hemidecorticated monkeys supports the view that lesions of higher cortical centers result in directional deficits. Tusa and collaborators ( $R$. J. Tusa, personal communication) observed profound pursuit deficits in the contralateral hemifield as expected from the loss of striate cortex but, in addition, observed a directionally asymmetric deficit in the intact hemifield with pursuit toward the side of the lesion being more severely damaged. In other words, a second, motor-related deficit seemed superimposed on the visual deficits that resulted from the striate cortex lesion. The cortical locus which gave rise to this motorrelated deficit has not yet been identified. The parietal lobe would seem a possible candidate for such function, but a recent report by Lynch and McLaren (1982) suggests that unilateral lesions of parietal and dorsal prestriate cortex in monkeys has little or no effect on pursuit, whereas bilateral lesions affect pursuit in both directions. Once again these monkeys were examined during steady-state foveal pursuit, and it is difficult to separate visual from motor effects under these conditions. Future experiments which combine more restricted cortical lesions with motion at controlled retinal loci should 

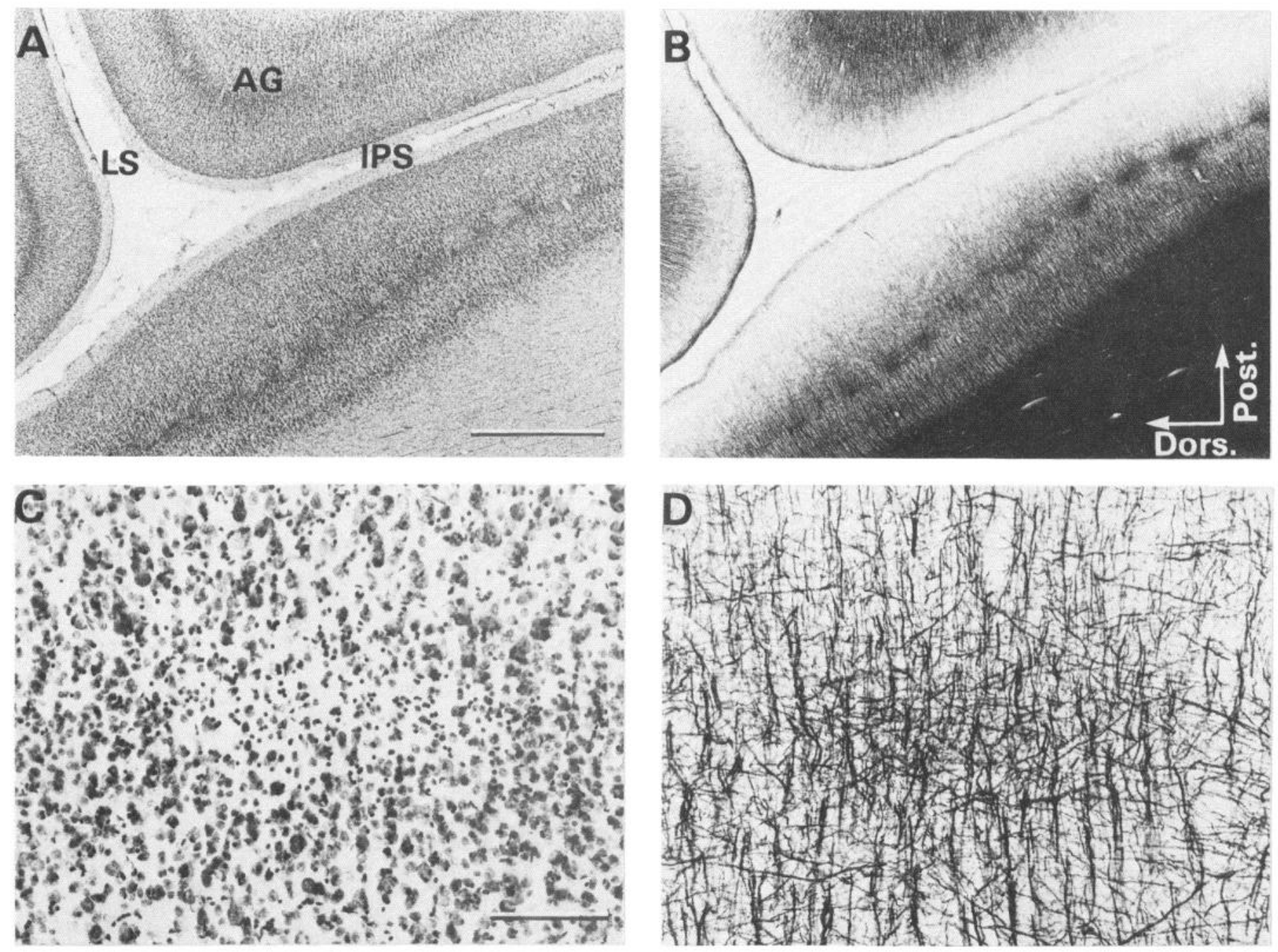

Figure 13. An unusual pathology observed in one monkey. Sections are in the parasagittal plane and show a region of buried cortex near the intersection of the lunate (LS) and intraparietal (IPS) sulci. A portion of the annectant gyrus ( $A G)$ is visible. The micrographs in $A$ and $B$ are oriented with dorsal to the left and posterior upward so that the pathological region of cortex can be conveniently viewed. $A$, The pathology appeared as a regular series of microlesions (average separation of 500 to $600 \mu \mathrm{m}$ ) visible as cell-sparse patches in layer IV of this cresyl violet-stained section. Calibration bar, $1.5 \mathrm{~mm}$. B, An adjacent section stained for myelinated fibers (Gallyas, 1979). The myelin-rich patches in the outer band of Baillarger superimpose directly on the cell-sparse patches in $A$ and presumably reflect the same underlying pathology. Calibration is the same as in $A$. C. High power micrograph of one of the microlesions in $A$. The lesion affects the upper two-thirds of layer IV and the lowest extent of layer III. Calibration bar, $120 \mu \mathrm{m}$. D, View of the same microlesion as in $C$ in an adjacent section stained for myelinated fibers. The myelin-dense region results from a proliferation of fibers rather than an increase in nonspecific background stain. Calibration is the same as in $C$.

clarify the nature of pursuit deficits that follow lesions of higher cortical areas. Our current hypothesis is that deficits following MT lesions result from disruption of visual processing, whereas the effects of lesions of higher cortical areas have motor components as well.

Recovery. The monkeys in our experiments recovered quickly from all of the behavioral deficits induced by the MT injections. A major question concerning the recovery is whether it is mediated by neural mechanisms within MT or by a cortical pathway parallel to MT. We assume that visual information mediating postlesion behavioral recovery must pass through visual cortex since unilateral striate lesions result in permanent pursuit initiation deficits of the type we have reported here (Goldberg et al., 1982). A limited number of physiological recordings suggest that surviving neurons near the border of our lesions have receptive fields which include the deficient portion of the visual field. We have not, in other words, eliminated the entire "point image" in MT of a particular region of visual space (Mcllwain, 1975). It is possible that such neurons could assume the functions of lost neurons within the damaged area. It is additionally possible that such neurons were temporarily inactivated by the ibotenic acid but recovered, since their exposure to the toxin was sublethal. The behavioral deficit might then reflect sublethal effects of ibotenic acid on a larger area of cortex than is seen in histological reconstruction. It seems likely that such a "halo" of reversibly inactivated neurons exists around the irreversibly damaged cortex, but gauging its size is difficult. Injections $\mathrm{C} 1$ and D1 were relatively peripheral in MT $\left(7^{\circ}\right.$ and $12^{\circ}$, respectively), and, in both, cases pursuit initiation was normal at $2^{\circ}$ and $5^{\circ}$ eccentric (Fig. 4, $B$ and C). Therefore, a behaviorally significant concentration of ibotenic acid did not reach the representation of the central $5^{\circ}$ of the visual field in MT. Judging from the histological reconstructions in Figure $12, A$ and $C$, and from published maps of the topographic representation of visual space in MT (Gattass and Gross, 1981; Van Essen et al., 1981), one can infer that behaviorally significant effects of the acid, even if reversible, are not likely to extend more than $3 \mathrm{~mm}$ from the center of the injection site.

Alternatively, behavioral recovery may be mediated by striatefugal pathways parallel to MT. The most likely pathway for cortical 
visual information to reach the pursuit system is via the dorsolateral pontine nuclei. These nuclei receive a major projection from MT (Maunsell and Van Essen, 1983c; Ungerleider, et al., 1984), contain numerous direction sclective neurons (Suzuki and Keller, 1984), and project in turn to pursuit-related centers of the cerebellum (Brodal, 1979, 1982; Langer et al., 1980). However, when horseradish peroxidase is injected into these nuclei, retrogradely filled neurons are observed not only in MT, but in other extrastriate areas as well (Glickstein et al., 1980). Other extrastriate areas are known to contain direction-selective neurons; the distinctive feature of MT lies in the very high proportion of such neurons that it contains. It is entirely possible, therefore, that alternative pathways from striate cortex to the pontine nuclei are able to provide sufficient information concerning visual motion for the pursuit system to operate adequately after loss of its inputs from MT.

The rapid recovery from the behavioral deficits raises the possibility that MT is not necessary for this particular function in a damaged nervous system (although this is still not certain, since the recovery may be mediated within MT). However, we do not believe that the recovery affects our basic conclusions regarding the role of MT in normal vision. In the past, transient deficits following surgical ablation have been rightly viewed with skepticism since the generalized trauma of a major surgical procedure might result in behavioral perturbations only distantly related to the specific functions of the removed cortex. General surgical trauma seems negligible in our experiments because the procedures were performed in awake monkeys with no signs of discomfort, the cortical lesions were very small, and a saline injection had no effect. Furthermore, the behavioral deficits were specific for a precise region of the visual field and for visual motion in particular. Such specificity militates against the notion that we are simply observing a general perturbation of the entire corlical visual system caused by the injection. The results suggest instead that the perturbation occurred in a cortical pathway that preferentially analyzes visual motion. Future experiments employing chemical lesions of MT in combination with psychophysical paradigms in which monkeys detect and report specific aspects of visual stimuli should provide firmer evidence concerning the specificity of neural processing in MT for motion. It is our long-term hope that such techniques may be employed in a behavioral analysis of function in other extrastriate areas as well.

\section{References}

Albright, T. D., R. Desimone, and C. G. Gross (1984) Columnar organization of directionally selective cells in visual area MT of the macaque. J. Neurophysiol. 51: 16-31.

Allman, J. M., and J. H. Kaas (1971) A representation of the visual field in the caudal third of the middle temporal gyrus of the owl monkey (Aotus trivirgatus). Brain Res. 31: 85-105.

Allman, J. M., J. H. Kaas, and R. H. Lane (1973) The middle temporal visual area (MT) in the bushbaby, Galago senegalensis. Brain Res. 57: 197-202.

Baker, J. F., S. E. Petersen, W. T. Newsome, and J. M. Allman (1981) Visual response properties of neurons in four extrastriate visual areas of the owl monkey (Aotus trivirgatus): A quantitative comparison of the medial, dorsomedial, dorsolateral, and middle temporal areas. J. Neurophysiol. 45: 397-416.

Brodal, P. (1979) The pontocerebellar projection in the rhesus monkey: An experimental study with retrograde axonal transport of horseradish peroxidase. Neuroscience 4: 193-208

Brodal, P. (1982) Further observations on the cerebellar projections from the pontine nuclei and the nucleus reticularis tegmenti pontis in the rhesus. $\mathrm{J}$. Comp. Neurol. 204: 44-55.

Cragg, B. G. (1969) The topography of the afferent projections in the circumstriate visual cortex of the monkey studied by the Nauta method. Vision Res. 9: 733-747.

Dubner, R., and S. M. Zeki (1971) Response properties and receptive fields of cells in an anatomically defined region of the superior temporal sulcus in the monkey. Brain Res. 35: 528-532.

Evarts, E. V. (1966) Methods for recording activity of individual neurons in moving animals. Methods Med. Res. 11: 241-250.

Evarts, E. V. (1968) A technique for recording activity of subcortical neurons in moving animals. Electroencephalogr. Clin. Neurophysiol. 24: 83-86.
Fischer, B., and R. Boch (1983) Saccadic eye movements after extremely short reaction times in the monkey. Brain Res. 260: 21-26.

Fuchs, A. F., and D. A. Robinson (1966) A method for measuring horizontal and vertical eye movement chronically in the monkey. J. Appl. Physiol. 21: 1068-1070.

Gallyas, F. (1979) Silver staining of myelin by means of physical development. Neurol. Res. 1: 203-209.

Gattass, R., and C. G. Gross (1981) Visual topography of striate projection zone (MT) in posterior superior temporal sulcus of the macaque. J. Neurophysiol. 46: 621-638.

Gizzi, M. S., W. T. Newsome, and J. A. Movshon (1983) Directional selectivity of neurons in macaque MT. Invest. Ophthalmol. Vis. Sci. Suppl. 24: 107.

Glickstein, M., J. L. Cohen, B. Dixon, A. Gibson, M. Hollins, E. LaBossiere, and F. Robinson (1980) Corticopontine visual projections in macaque monkeys. J. Comp. Neurol. 190: 209-229.

Goldberg, M. E., C. J. Bruce, L. Ungerleider, and M. Mishkin (1982) Role of the striate cortex in generation of smooth pursuit eye movements. Ann. Neurol. 12: 113.

Guldin, W. O., and H. J. Markowitsch (1981) No detectable remote lesions following massive intrastriatal injections of ibotenic acid. Brain Res. 225: 446-451.

Hays, A. V., B. J. Richmond, and L. M. Optican (1982) A UNIX-based multiple process system for real-time data acquisition and control. WESCON Conference Proceedings, Session 2, No. 1: 1-10.

Hikosaka, O., and R. H. Wurtz (1983) Visual and oculomotor functions of monkey substantia nigra pars reticulata. I. Relation of visual and auditory responses to saccades. J. Neurophysiol. 49: 1230-1253.

Judge, S. J., B. J. Richmond, and F. C. Chu (1980) Implantation of magnetic search coils for measurement of eye position: An improved method. Vision Res. 20: 535-538.

Langer, T. P., A. F. Fuchs, M. C. Chubb, and C. Scudder (1980) Afferent projections to the monkey flocculus. Soc. Neurosci. Abstr. 6: 477 .

Lanman, J., E. Bizzi, and J. Allum (1978) The coordination of eye and head movement during smooth pursuit. Brain Res. 153: 39-53.

Lisberger, S. G., and L. E. Westbrook (1985) Properties of visual inputs that initiate horizontal smooth pursuit eye movements in monkeys. J. Neurosci., in press.

Lynch, J. C., and J. W. McLaren (1982) The contribution of parieto-occipital association cortex to the control of slow eye movements. In Functional Basis of Ocular Motility Disorders, D. S. Zee and E. L. Keller, eds., pp. 501-510, Pergamon Press, Oxford.

Maunsell, J. H. R., and D. C. Van Essen (1983a) Functional properties of neurons in the middle temporal visual area of the macaque monkey. I. Selectivity for stimulus direction, speed, and orientation. J. Neurophysiol. 49: $1127-1147$.

Maunsell, J. H. R., and D. C. Van Essen (1983b) Functional properties of neurons in middle temporal visual area of the macaque monkey. II. Binocular interactions and sensitivity to binocular disparity. J. Neurophysiol. 49: $1148-1167$

Maunsell, J. H. R., and D. C. Van Essen (1983c) The connections of the middle temporal visual area (MT) and their relationship to a cortical hierarchy in the macaque monkey. J. Neurosci. 3: 2563-2586.

Mcllwain, J. T. (1975) Visual receptive fields and their images in superior colliculus of the cat. J. Neurophysiol. 38: 219-230.

Miezin, F., E. McGuinness, and J. Allman (1982) Antagonistic directionspecific mechanisms in area MT in the owl monkey. Soc. Neurosci. Abstr. 8: 681 .

Mikami, A., W. T. Newsome, and R. H. Wurtz (1983) Interaction distances for successively flashed stimuli in macaque MT. Soc. Neurosci. Abstr. 9: 477.

Mohler, C. W., and R. H. Wurtz (1977) Role of striate cortex and superior colliculus in visual guidance of saccadic eye movements in monkeys. $\mathrm{J}$. Neurophysiol. 40: 74-94.

Movshon, J. A., E. H. Adelson, M. S. Gizzi, and W. T. Newsome (1984) The analysis of moving visual patterns. Exp. Brain Res., in press.

Newsome, W. T., and R. H. Wurtz (1981) Response properties of single neurons in the middle temporal visual area (MT) of alert macaque monkeys. Soc. Neurosci. Abstr. 7: 832

Newsome, W. T., R. H. Wurtz, M. R. Dürsteler, and A. Mikami (1983) Deficits in pursuit eye movements following chemical lesions of motion-related visual areas in the superior temporal sulcus of the macaque monkey. Soc. Neurosci. Abstr. 9: 154.

Newsome, W. T., R. H. Wurtz, M. R. Dürsteler, and A. Mikami (1985) Punctate chemical lesions of striate cortex in the macaque monkey: Effect on visually guided saccades. Exp. Brain Res., in press. 
Rabiner, L. R., and B. Gold (1975) Theory and Application of Digital Signal Processing, Chap 3. Prentice-Hall, Englewood Cliffs, NJ.

Rashbass, C. (1961) The relationship between saccadic and smooth tracking eye movements. J. Physiol. (Lond.) 159: 326-338.

Robinson, D. A. (1963) A method of measuring eye movement using a scleral search coil in a magnetic field. IEEE Trans. Biomed. Eng. 10: 137145.

Robinson, D. A. (1981) Control of eye movements. In Handbook of Physiology. Sect. 1: The Nervous System. Vol. II: Motor Control, Part 2, V. B. Brooks, ed., pp. 1275-1320, American Physiological Society, Bethesda, MD.

Schwarcz, R., T. Hokfelt, K. Fuxe, G. Jonsson, M. Goldstein, anıd L. Terenius (1979) Ibotenic acid-induced neuronal degeneration: A morphological and neurochemical study. Exp. Brain Res. 37: 199-216.

Segraves, M. A., S. -Y. Deng, C. J. Bruce, L. G. Ungerleider, M. Mishkin, and M. E. Goldberg (1983) Monkeys with striate lesions have saccadic dysmetria to moving targets. Invest. Ophthalmol. Vis. Sci. Suppl. 24: 25.

Sparks, D. L., R. Holland, and B. L. Guthrie (1976) Size and distribution of movement fields in the monkey superior colliculus. Brain Res. 113: 2134.

Spatz, W. B., and J. Tigges (1972) Experimental-anatomical studies on the "middle temporal visual area (MT)" in primates. I. Efferent corticocortical connections in the marmoset Callithrix jacchus. J. Comp. Neurol. 146: 451-464.

Spatz, W. B., and J. Tigges (1973) Studies of the visual area MT in primates. II. Projection fibers to subcortical structures. Brain Res. $61: 374-378$.

Standage, G. P., and L. A. Benevento (1983) The organization of connections between the pulvinar and visual area MT in the macaque monkey. Brain Res. 262: 288-294.

Suzuki, D. A., and E. L. Keller (1984) Visual signals in the dorsolateral pontine nucleus of the alert monkey: Their relationship to smooth-pursuit eye movements. Exp. Brain Res. 53: 473-478.

Tigges, J., M. Tigges, S. Anschel, N. A. Cross, W. D. Letbetter, and R. L. McBride (1981) Areal and laminar distribution of neurons interconnecting the central, visual cortical areas $17,18,19$ and MT in the squirref monkey (Saimiri). J. Comp. Neurol. 202: 539-560.

Troost, B. T., and L. A. Abel (1982) Pursuit disorders. In Functional Basis of Ocular Motility Disorders, G. Lennerstrand, D. S. Zee, and E. L. Keller, eds., pp. 511-515, Pergamon Press, Oxford.

Troost, B. T., R. B. Daroff, R. B. Weber, and L. F. Dell'Osso (1972) Hemispheric control of eye movements. II. Quantitative analysis of smooth pursuit in a hemispherectomy patient. Arch. Neurol. 27: 449-452.

Ungerleider, L. G., and M. Mishkin (1979) The striate projection zone in the superior temporal sulcus of Macaca mulatta: Location and topographic organization. J. Comp. Neurol. 188: 347-366.
Ungerleider, L. G., R. Desimone, and M. Mishkin (1982) Cortical projections of area MT in the macaque. Soc. Neurosci. Abstr. 8: 680 .

Ungerleider, L. G., R. Desimone, T. W. Galkin, and M. Mishkin (1984) Subcortical projections of area MT in the macaque. J. Comp. Neurol. 223 368-386.

Van Essen, D. C. (1979) Visual areas of the mammalian cerebral cortex. Annu. Rev. Neurosci. 2: 227-263.

Van Essen, D. C., and J. H. R. Maunsell (1980) Two-dimensional maps of the cerebral cortex. J. Comp. Neurol. 191: 255-281.

Van Essen, D. C., J. H. R. Maunsell, and J. L. Bixby (1981) The middle temporal visual area in the macaque: Myeloarchitecture, connections, functional properties and topographic organization. J. Comp. Neurol. 199: 293-326.

Weller, R. E., and J. H. Kaas (1983) Retinotopic patterns of connections of area 17 with visual areas, V-II and MT in macaque monkeys. J. Comp. Neurol. 220: 253-279.

Weller, R. E., J. Wall, and J. H. Kaas (1984) Cortical connections of middle temporal visual area (MT) and the superior temporal cortex in owl monkey. J. Comp. Neurol., 228: 81-104.

Wohlbarsht, M. L., E. F. MacNichol, Jr., and H. G. Wagner (1960) Glass insulated platinum microelectrode. Science 132: $1309-1310$.

Woolsey, C. N. (1981) Cortical Sensory Organization. Vol. 2: Multiple Visual Areas, Humana Press, Clifton, NJ.

Wurtz, R. H. (1969) Visual receptive fields of striate cortex neurons in awake monkeys. J. Neurophysiol. 32: 727-742.

Wurtz, R. H. A. Mikami, W. T. Newsome, and M. R. Dürsteler (1984a) Visual motion processing for perception and movement in the monkey cerebral cortex. In Perspectives of Neuroscience: From Molecule to Mind, $\mathrm{Y}$. Tsukada, ed., pp. 255-273. Tokyo University Press, Tokyo, in press.

Wurtz, R. H., B. J. Richmond, and W. T. Newsome (1984b) Modulation of cortical visual processing by attention, perception, and movement. In Dynamic Aspects of Neocortical Function, G. M. Edelman, W. M. Cowan, and W. E. Gall, eds., pp. 195-217, John Wiley \& Sons, Inc., New York.

Zee, D. S., P. H. Butler, L. M. Optican, R. J. Tusa, and G. Gucer (1982) Effects of bilateral occipital lobectomies on eye movements in monkeys: Preliminary observations. Doc. Ophthalmol. Proc. Ser. 34: 225-232.

Zeki, S. M. (1969) Representation of central visual fields in prestriate cortex of monkey. Brain Res. 14: 271-291.

Zeki, S. M. (1974a) Functional organization of a visual area in the posterior bank of the superior temporal sulcus of the rhesus monkey. J. Physiol. (Lond.) 236: 549-573.

Zeki, S. M. (1974b) Cells responding to changing image size and disparity in the cortex of the rhesus monkey. J. Physiol. (Lond.) 242: 827-841.

Zeki, S. M. (1978) Functional specialization in the visual cortex of the rhesus monkey. Nature 274: 423-428.

Zihl, J., D. von Cramon, and N. Mai (1983) Selective disturbance of movement vision after bilateral brain damage. Brain 106: 313. 